\title{
Population balance modelling and laser diagnostic validation of soot particle evolution in laminar ethylene diffusion flames
}

\author{
Anxiong Liu ${ }^{a}$. Carlos E. Garcia ${ }^{a}$, Fabian Sewerin ${ }^{a, b}$, Benjamin A.O. Williams ${ }^{a, c}$, Stelios \\ Rigopoulos ${ }^{a}$ † \\ ${ }^{a}$ Department of Mechanical Engineering, Imperial College London, Exhibition Road, London SW7 2AZ, UK \\ ${ }^{b}$ Institute of Solid Mechanics, Technische Universität Braunschweig, Langer Kamp 8, 38106 Braunschweig, \\ Germany \\ ${ }^{c}$ Department of Engineering Science, University of Oxford, Parks Road, Oxford OXI 3PJ, UK
}

\begin{abstract}
Laminar diffusion flames present an elementary configuration for investigating soot formation and validating kinetic models before these are transferred to turbulent combustors. In the present article, we present a joint experimental and modelling investigation of soot formation in a laminar co-flow burner. The diffusion flames are analysed with the aid of laser diagnostic techniques, including elastic light scattering (ELS), planar laser-induced fluorescence of $\mathrm{OH}$ (OH-PLIF) and line-of-sight attenuation (LOSA), to measure the spatial distribution of soot, gas phase species and the line-of-sight integrated soot volume fraction (ISVF), respectively. The experimental dataset is supplemented by location-specific TEM images of thermophoretically sampled soot particles. The simulation of the sooting flames is carried out with a recently developed discretisation method for the population balance equation (Liu and Rigopoulos, 2019, Combust. Flame 205, 506-521) that accomplishes an accurate prediction of the particle size distribution, coupled with an in-house CFD code. By minimising numerical errors, we ensure that the discrepancies on the modelling side are mainly due to kinetics and are able to carry out an investigation of alternative models. We include a complete set of soot kinetics for PAH-based nucleation and condensation, HACA-based surface growth and oxidation as well as size-dependent aggregation, and consider three different gas phase reaction mechanisms (ABF, BBP and KM2). Based on predictions of the gas phase composition and particle size distribution of soot, modelled counterparts of the laser diagnostic signals are computed and compared with the experimental measurements. The approach of directly predicting signals circumvents the difficulties of explicitly representing the $\mathrm{OH}$ concentration in terms of the measured OH-PLIF data and avoids using 'hybrid' modelled and measured values to approximate the $\mathrm{OH}$ concentration. Moreover, the LOSA signal is directly converted to the line-of-sight ISVF instead of a measure of local soot volume fraction to avoid tomographic inversion errors. Lastly, the predicted ELS signal is computed in terms of the particle size distribution resolved by the population balance model, thus circumventing the approximation of an integral soot property using a presumed size distribution. While we cannot obtain quantitative agreement between experiments and simulations, the accuracy of the numerical approach and the direct prediction of experimental signals allow us to conduct sensitivity analyses of key empirical parameters and investigate the importance of the PAH chemistry and its influence on the competition between nucleation, condensation and surface growth.
\end{abstract}

Keywords: Soot formation, Planar laser-induced fluorescence (PLIF), Elastic light scattering (ELS), Line-of-sight attenuation (LOSA), Population balance, Sectional method, Laminar flame

\section{Introduction}

Soot is composed of polydispersed particles that form in the process of hydrocarbon combustion. Soot is a major air pollutant with severe impacts on human health and, furthermore, affects combustion efficiency and safety as it can accumulate inside combustors or their components. On the other hand, soot particles enhance the radiative heat transfer and are thus sought after in equipment such as furnaces. The impact of the smaller soot particles on human health is being increasingly acknowledged (see, e.g., Reference [39]) and new regulations are imposed on

\footnotetext{
${ }^{*}$ Present address: Department of Mechanical Engineering, University College London, Torrington Place, London WC1E 7JE, UK

${ }^{\dagger}$ Corresponding author (s.rigopoulos@imperial.ac.uk)
} 
the number and size of emitted soot particles, thus placing increasing emphasis on the prediction and control of the soot particle size distribution. The spatial and temporal evolution of the distribution can be described by the population balance equation (PBE). For a review of the PBE with emphasis on its application to soot modelling, we refer to References [55, 53, 56]. Models of soot formation in flames must combine the PBE with computational fluid dynamics (CFD) and models for gas phase chemistry and soot formation processes, which include nucleation, condensation, surface growth, oxidation and aggregation.

In the past two decades, our physical understanding of the fundamental mechanisms of soot formation has consistently increased. In a first step, macro-molecular polycyclic aromatic hydrocarbons (PAHs) are thought to coalesce into soot precursors and nascent soot particles [35]. Second, acetylene $\left(\mathrm{C}_{2} \mathrm{H}_{2}\right)$ plays an important role in increasing the hydrocarbon content of soot particles and driving surface growth. These acetylene-based descriptions [21, 46, 43, 77], including the Hydrogen-Abstraction- $\mathrm{C}_{2} \mathrm{H}_{2}$-Addition (HACA) mechanism [33, 6, 9, 52, 16, 73], are extensively applied and well-established by now. Third, the hydroxyl radical $(\mathrm{OH})$ and molecular oxygen $\left(\mathrm{O}_{2}\right)$ are the most effective species for oxidising soot particles. Despite agreement on these general features, a number of controversies remain regarding the model formulation for particle inception and the calibration or adjustment of kinetic parameters. For example, nucleation is commonly related to the collision of PAH species [6, 9, 52, 73], but since nascent soot can display various different morphologies [70, 35], several PAH dimerisation models have been proposed [23, 6, 9, 73, 75]. However, detailed kinetic descriptions of PAH growth prior to inception involve many parameters which cannot be measured directly and need to be identified based on a limited number of measured integral quantities. As a second example, we mention the fraction of active sites $\alpha$ in the HACA mechanism which is often taken as constant for a particular flame configuration [22, 16]. Since experimental observations on particle surface ageing point towards a decrease in the fraction of active sites, $\alpha$ has, alternatively, been represented as a function of particle size [6], local flame temperature [20, 26] or particle residence time [68]. However, the coefficients in the kinetic expressions for $\alpha$ that were calibrated based on experimental data from different flames and for use with different reaction mechanisms vary widely.

Future efforts for successfully modelling sooting flames rely on a moderately detailed physical description, an accurate numerical solution method and a careful and well-coordinated validation step at a clearly defined interface of predicted and measured quantities, minimising the mutual amplification of experimental uncertainties. In the present article, we aim at contributing to these aspects in three main ways. First, by solving the discretised PBE using a new and very accurate discretisation method [45], the cancellation of numerical and modelling errors is avoided, preparing the ground for an analysis of different kinetic models. Second, by predicting directly the experimental signals, we circumvent several uncertainties and implicit assumptions related to the conversion of signals. Third, by virtue of the above two points, a parametric study of the influence of gas phase and soot kinetics on the soot formation and distribution in laminar diffusion flames is conducted.

Concomitantly, we report on a new set of laser diagnostic measurements on a laminar co-flow diffusion flame, supplemented by the thermophoretic sampling of soot particles. In particular, planar laser-induced fluorescence of $\mathrm{OH}(\mathrm{OH}-\mathrm{PLIF})$ is used to provide data for validating chemical kinetics, elastic light scattering (ELS) provides insight into the spatial distribution of soot and line-of-sight attenuation (LOSA) measurements allow us to determine the integrated soot volume fraction (ISVF). Location-specific samples are extracted with the aid of a thermophoretic sampling device according to Dobbins and Megaridis [15], shedding light on the morphology of soot aggregates.

The method employed for the numerical solution of the PBE is a recently published finite volume method for the direct solution of the PBE [45]. The objective of this method is to accomplish an accurate prediction of the particle size distribution on an arbitrary non-uniform grid in the particle volume domain, while conserving the soot volume fraction during particle aggregation. By minimising numerical errors, we ensure that the uncertainties on the modelling side are mainly of a physical nature, relating to gas phase and soot formation kinetics, thus supporting a systematic investigation of alternative kinetics. The method is coupled with our in-house CFD code BOFFIN [32]; the coupling of the method with CFD has been previously tested and validated [45].

In order to minimise the accumulation of experimental uncertainties, we adopt the calibration/validation paradigm advocated by Connelly et al. [13] and compare experimental laser diagnostic signals with predicted signals computed from the modelled gas phase composition and soot particle size distribution. This approach is advantageous as it circumvents several assumptions that are commonly made in converting experimental laser diagnostic signals into physically meaningful quantities. One of these assumptions is related to the experimental OH-PLIF signal, which is determined not only by the local $\mathrm{OH}$ concentration, but also by temperature and by collisional quenching owing to the presence of other species. When inferring the $\mathrm{OH}$ concentration from an OH-PLIF signal, the temperature and concentrations of other species are often approximated by predicted values and the dependence of the collisional quenching rate on $\mathrm{OH}$ is omitted, leading, strictly, to $\mathrm{OH}$ concentration reports that 
are a mixture of measured and modelled data. The spatial distribution of soot volume fraction, moreover, is often reconstructed from LOSA measurements using a tomographic inversion which incurs an error accumulation inbetween the flame edges and the centreline [64]. In a signal-based comparison, by contrast, the experimental and predicted data remain strictly separate, whence the signals constitute an apt interface for calibration and validation. The prediction of the ELS signal presents another challenging case because it constitutes a statistical property of the particle size distribution. In comparisons with model predictions, the ELS signal is typically converted into a measure of mean particle size by assuming a particular shape of the soot size distribution. Since, in our PBE-based approach, the size distribution is accurately resolved, the ELS signal can be directly computed without such an assumption.

Drawing on a signal-based comparison, the influence of the gas phase reaction mechanism and the corresponding soot nucleation/condensation kinetics is evaluated for three well-established reaction mechanisms (ABF, BBP and KM2). Here, we include a complete set of soot kinetics for PAH-based nucleation and condensation, HACAbased surface growth and oxidation as well as size-dependent aggregation. Sensitivity analyses in terms of key empirical parameters in the soot nucleation, condensation and surface growth kinetics are undertaken to elucidate potential causes for the observed discrepancies and deduce practical guidelines for tuning the soot model. While it must be acknowledged that quantitative agreement between simulations and experiments for all soot variables is not possible at the moment, we hope that the analysis in this article will contribute to an understanding of the issues underlying the discrepancies.

This article is organised as follows. In Section 2 , we briefly summarise the flame configurations investigated here and relay details on the experimental measurement techniques. Subsequently, in Section 3, the physical model for a reacting multicomponent gas is presented and the PBE-based soot model is discussed. Here, we provide details on the evaluation of the kinetic rates which affect the soot particle size distribution and lay emphasis on an account of soot morphology. The computation of laser-diagnostic signals in terms of the gas phase composition and soot particle size distribution is addressed in Section 4, before we assess the physical model by comparing predicted and measured laser-diagnostic signals in Section 5 . Concomitantly, the influence of the gas phase chemistry on the spatial distribution of soot precursors and key kinetic rates is elucidated. This is followed by a summary of the main observations in Section 6 .

\section{Experimental approach}

The Santoro flame [58] constitutes a geometrically simple and reproducible standard configuration for investigating soot formation and for model validation. The co-flow diffusion burner consists of a nozzle with an internal diameter of $11.1 \mathrm{~mm}$ protruding $4 \mathrm{~mm}$ above a $101.6 \mathrm{~mm}$ diameter co-flow tube (Figure 1). The flame is shielded from ambient air currents by a $400 \mathrm{~mm}$ tall chimney. Following Santoro et al. [58, 59], we investigate the same 'nonsmoking' and 'incipient-smoking' cases with fuel flow velocities of $3.98 \mathrm{~cm} / \mathrm{s}$ and $4.75 \mathrm{~cm} / \mathrm{s}$, respectively, and an air flow velocity of $8.90 \mathrm{~cm} / \mathrm{s}$ for both. Mass flow controllers (Bronkhorst EL-FLOW series, nominal accuracy $1.0 \%$ ) are used to meter the gases to match Santoro's conditions within experimental error. The accuracy of the mass flow controllers was also verified by cross-checking against all such meters available in the laboratory. Three non-intrusive laser diagnostic techniques are employed: ELS, OH-PLIF and LOSA. In addition, soot particles are thermophoretically sampled from the flame and their morphologies are analysed using transmission electron microscopy (TEM).

For the ELS measurements, a Q-switched frequency-doubled Nd:YAG laser with four independent heads is used in conjunction with sheet-forming optics to illuminate a $23 \mathrm{~mm} \times 400 \mu \mathrm{m}$ region across the burner centreline (Figure 2). The ELS signal is collected using a CCD camera with a $105 \mathrm{~mm} \mathrm{f} / 2.8$ lens placed perpendicular to the laser beam with an exposure time of $1 \mu \mathrm{s}$. A narrow bandpass filter centred around $532 \mathrm{~nm}$ is used to reject light originating from inelastic processes. The laser pulse energy is approximately $0.5 \mathrm{~mJ}$ and the repetition rate is $10 \mathrm{~Hz}$. Each image frame is based on 600 instantaneous exposures from which the mean and standard deviation are computed. Measurements at different heights are obtained by translating the burner vertically using a micrometer stage.

The Nd:YAG laser is further used to pump a frequency-doubled Rhodamine $6 \mathrm{G}$ dye laser tuned to $283.6 \mathrm{~nm}$ to excite the $Q_{1}(8)(v=1 \leftarrow 0)$ transition in the $A^{2} \Sigma \leftarrow X^{2} \Pi$ electronic system of the $\mathrm{OH}$ radical. This transition has been chosen in order to reduce the sensitivity of the LIF signal to temperature. The fluorescence signals from the $(v=0 \leftarrow 0)$ and $(v=1 \leftarrow 1)$ branches are collected in the wavelength range from $309 \mathrm{~nm}$ to $315 \mathrm{~nm}$ with an intensifier ( $80 \mathrm{~ns}$ gate) and a CCD camera. A long-pass filter is used to suppress the $283 \mathrm{~nm}$ incident Rayleigh wavelength (in addition to gating out the incident pulse). Strong broadband fluorescence attributed to $\mathrm{PAH}$ species is detected in addition to fluorescence from the $\mathrm{OH}$ radical. The two fluorescence signals are separated 
by subtracting the signal detected when the laser is detuned from the $\mathrm{OH}$ spectral line from the signal recorded when the laser is tuned $\left(S^{\mathrm{OH}}=S_{283.6 \mathrm{~nm}}^{\text {Total }}-S_{284.0 \mathrm{~nm}}^{\mathrm{PAH}}\right)$. The UV laser sheet is $700 \mu \mathrm{m}$ thick and $58 \mathrm{~mm}$ tall to avoid exceeding the $\mathrm{OH}$ saturation intensity $\left(10 \mathrm{~mJ} / \mathrm{cm}^{2}\right.$ [60] ) with a shot energy of no more than $3.5 \mathrm{~mJ}$, thus ensuring operation within the linear LIF regime. In an unrelated experiment using the same set-up [47], the OH-PLIF signal was found to correlate linearly with the concentration of silane doping (0-3000 ppm) added to a vitiated co-flow burner with an $R^{2}$ coefficient of 0.9994 , further confirming linearity.

The ELS and LIF signals are post-processed as follows. First, a dark image obtained immediately prior to the experiment is subtracted. Subsequently, relative intensity variations along the vertical axis are corrected using a measurement of the beam profile recorded immediately prior to the experiment. Normalised beam profiles are obtained by imaging a quartz cuvette placed on the optical path above the burner, containing a dilute solution of milk in water (green beam) or acetone vapour (UV beam). Next, the intensity of each individual image frame is scaled according to shot energy measurements from a PEM-45K energy meter (green beam) and a LaVision energy monitor (UV beam) acquired during the experiment for each laser shot. The spatial coordinate transformation between image and experiment was established by imaging a double-sided dot target ( $1 \mathrm{~mm}$ diameter, $2.5 \mathrm{~mm}$ pitch) that attaches to the burner nozzle and applying the de-warping algorithm available in the LaVision DaVis package. A script was used to discard any image in which the sooty wings of the flame were detected to be displaced by more than $\pm 450 \mu \mathrm{m}$, e.g., due to an air draft.

The LOSA data are obtained by scanning a $635 \mathrm{~nm}$ diode laser across the flame in increments of $250 \mu \mathrm{m}$ with a power of $1.2 \mathrm{~mW}$ and focussed down to a diameter of $0.5 \mathrm{~mm}$ over the burner. The transmitted power is measured with a $0.8 \mathrm{~mm}^{2} \mathrm{Si}$ photodetector. A digital oscilloscope is used to read voltage from the photodetector and obtain measurements averaged over one second. The extinction coefficient is calculated using as reference the voltage corresponding to an unobstructed beam and subtracting the voltage obtained when the beam is fully obstructed. Extinction due to air and laser power variations were both found to be negligible over the path length and the duration of the experiment, respectively.

Furthermore, we employ a thermophoretic sampling device to extract soot particles from the flames. The device consists of a double-action pneumatic cylinder that introduces a $3 \mathrm{~mm}$ diameter holey carbon TEM grid into the flame, retracting after a pre-set residence time of $120 \mathrm{~ms}$. The grid is held vertically during sampling in order to keep disruption of the flow to a minimum. Samples are imaged in a Jeol 2100+ TEM at an acceleration potential of $200 \mathrm{kV}$. Quantitative data on the size of primary particles is obtained from the analysis of a collection of 435 TEM images. The distinction between individual particles in TEM images is difficult because several particles may be on top of each other. In order to avoid errors from an automated algorithm, a total of 762 primary particles were manually measured with Image-J, using the scale bar on the image as calibration. The mean particle diameter, standard deviation and a rough particle size distribution were then computed for each case and height above the burner.

\section{Physical model}

\subsection{Gas phase transport and chemistry}

Figure 1 schematically depicts the axisymmetric, two-dimensional domain we consider for modelling the diffusion flame on the Santoro burner. Symmetry boundary conditions are applied along the flame centreline, while Dirichlet boundary conditions are imposed along the lateral domain boundary and homogeneous Neumann boundary conditions are applied at the outlet. At the inflow cross-section, Dirichlet boundary conditions hold. Initially, the domain contains only air at ambient pressure and temperature; starting from this initial condition, the governing equations are temporally evolved into steady-state. The spatial grid encompasses $200 \times 100$ cells in the axial and radial directions, respectively, and is refined towards the jet exit and the interface between the fuel and air streams. Following common practice, we describe the gas phase in terms of $n+1$ reactive scalars $\mathbf{Y}(\mathbf{x}, t)=\left(Y_{1}(\mathbf{x}, t), \ldots, Y_{n}(\mathbf{x}, t), Y_{n+1}(\mathbf{x}, t)\right)$, representing $n$ species mass fractions and the mixture enthalpy. Within the flow, the reactive scalars evolve according to the species transport equations and the energy conservation equation, respectively, and obey the constitutive relations for a multicomponent ideal gas. The flow field, moreover, is governed by the continuity and momentum conservation equations for a Newtonian fluid.

In order to support the PAH-HACA framework for soot formation, three different chemical reaction mechanisms are considered. The first one is the ABF mechanism which encompasses 101 species and 544 chemical reactions [71, 6]. Here, the chemistry for small hydrocarbons is based on the GRI 1.2 mechanism which contributes most $\mathrm{C}_{2}$ species and a skeletal mechanism for $\mathrm{C}_{3}$ and $\mathrm{C}_{4}$ species. The $\mathrm{ABF}$ mechanism includes PAH species up to pyrene $\left(\mathrm{C}_{16} \mathrm{H}_{10}\right.$ or $\left.\mathrm{A}_{4}\right)$, in particular, benzene, naphthalene, phenanthrene and pyrene $\left(\mathrm{A}_{1}-\mathrm{A}_{4}\right)$. The $\mathrm{ABF}$ mech- 
anism also serves as a reference for other reaction mechanisms [16, 72] used in modelling soot formation .

The second chemical reaction mechanism we consider is provided by Blanquart et al. [8] (BBP), involving 149 species and 928 reactions, and accounts for all major pathways of PAH formation up to cyclopentapyrene $\left(\mathrm{C}_{18} \mathrm{H}_{10}\right)$. The BBP mechanism has been developed based on GRI 3.0 [19] with an emphasis on the chemistry of soot precursors such as acetylene, the $\mathrm{C}_{3} \mathrm{H}_{4}$ isomers (allene and propyne), propene, butadiene and benzenel. The complete mechanism has been validated for a large set of fuels ranging from methane to iso-octane and one-ring aromatics for configurations including homogeneous auto-ignition, laminar premixed flames and diffusion flames.

The last reaction mechanism is the KAUST PAH Mechanism 2 (KM2) [72] which includes species up to coronene $\mathrm{C}_{24} \mathrm{H}_{12}$ and comprises of 202 species and 1351 reactions in total. The reactions for small chemical species up to benzene were adopted from the USC II mechanism. Predictions of PAH concentrations were validated in several premixed flames. In addition, Lee et al. [41] compared predictions of large PAH species with signals from a laser-induced incandescence (LII) experiment, indicating that it is well suited for modelling large PAH molecules that serve as soot precursors.

The thermodynamic and transport properties of the species are obtained from the ABF, BBP and KM 2 mechanisms, respectively, and a mixture-averaged diffusion coefficient is employed [2]. In the momentum equation, moreover, buoyancy effects are included. In order to account for the reduction of mixture enthalpy due to radiation by gas phase molecules and soot, we include a radiation model based on the assumption of optical thinness [40]. Here, the temperature dependence of the Planck mean absorption coefficients is approximated by polynomials [1].

\subsection{Population balance model for soot}

In a continuum approach, soot is commonly described in terms of the number of particles per unit of volume in physical space whose characteristic properties take on particular values. Here, we specifically consider particle volume $v$ as the single defining property; the number density $n(v, \mathbf{x}, t) \mathrm{d} v$ then corresponds to the number of particles with volume in $[v, v+\mathrm{d} v]$ at the location $\mathbf{x}$ and time $t$ per unit of physical space. Formally, the population balance equation (PBE) is a statement on the balance of number density in $(v, \mathbf{x})$-space, respecting source and sink processes for the creation and removal of number density [30]. At $(\mathbf{x}, t), n(\cdot, \mathbf{x}, t)$ is often referred to as the particle size distribution. Considering soot nucleation and aggregation as source/sink processes, the PBE is given by

$$
\begin{array}{r}
\frac{\partial n(v, \mathbf{x}, t)}{\partial t}+\sum_{j=1}^{3} \frac{\partial\left(\left(u_{j}+U_{j}^{\mathrm{T}}\right) n(v, \mathbf{x}, t)\right)}{\partial x_{j}}+\frac{\partial(G(v, \mathbf{Y}) n(v, \mathbf{x}, t))}{\partial v}=-\sum_{j=1}^{3} \frac{\partial K_{j}(v, \mathbf{x}, t)}{\partial x_{j}}+\dot{B}(\mathbf{Y}) \delta\left(v-v_{0}\right) \\
\quad+\frac{1}{2} \int_{0}^{v} \beta(v-w, w) n(v-w, \mathbf{x}, t) n(w, \mathbf{x}, t) \mathrm{d} w-\int_{0}^{\infty} \beta(v, w) n(v, \mathbf{x}, t) n(w, \mathbf{x}, t) \mathrm{d} w, \quad(1)
\end{array}
$$

where $G(v, \mathbf{Y})$ is the combined surface growth and oxidation rate, $\dot{B}$ represents the nucleation rate, $v_{0}$ is the nuclei volume and $\beta(v, w)$ denotes the aggregation kernel. $K_{j}(v, \mathbf{x}, t)$, moreover, represents the diffusive flux of number density along the $j$ th coordinate direction in physical space,

$$
K_{j}(v, \mathbf{x}, t)=-D_{\mathrm{p}}(v, \mathbf{x}, t) \frac{\partial n(v, \mathbf{x}, t)}{\partial x_{j}},
$$

and $D_{\mathrm{p}}(v, \mathbf{x}, t)$ represents the kinematic diffusivity of a particle with volume $v[24] . D_{\mathrm{p}}(v, \mathbf{x}, t)$ is controlled by the diameter $d_{\mathrm{p}}(v)$ of spherical particles, or the hydrodynamic diameter $d_{\mathrm{h}}(v)$ for aggregates, as indicated in investigations on the dynamic properties of polymers suspended in a solvent [37].

In the spatial convection term of Eq. (1), $\mathrm{U}^{\mathrm{T}}(v, \mathbf{x}, t)$ represents the thermophoretic velocity that a particle immersed in a flow with temperature gradients experiences. Depending on the Knudsen number Kn, the thermophoretic velocity is computed for the free-molecular $(\mathrm{Kn}>10)$, transitional $(0.1<\mathrm{Kn}<10)$ or continuum regimes $(\mathrm{Kn}<0.1)$ [69, 11, 62],

$$
U_{j}^{\mathrm{T}}=\left\{\begin{array}{ll}
-\frac{3 \nu}{4\left(1+\frac{\pi \alpha_{\mathrm{m}}}{8}\right)} \frac{1}{T} \frac{\partial T}{\partial x_{j}}, & 10<\mathrm{Kn} \\
-\frac{2 C_{\mathrm{s}} \nu\left(\frac{k_{\mathrm{g}}}{k_{\mathrm{p}}}+C_{\mathrm{t}} \mathrm{Kn}\right)}{\left(1+2 C_{\mathrm{m}} \mathrm{Kn}\right)\left(1+\frac{2 k_{\mathrm{g}}}{k_{\mathrm{p}}}+2 C_{\mathrm{t}} \mathrm{Kn}\right)} \frac{1}{T} \frac{\partial T}{\partial x_{j}}, & 0.1<\mathrm{Kn}<10 \\
0, & \mathrm{Kn}<0.1
\end{array},\right.
$$


where the accommodation factor $\alpha_{\mathrm{m}}$ is set to $0.9 . k_{\mathrm{g}}$ represents the thermal conductivity of the ambient gas and $k_{\mathrm{p}}=0.26 \mathrm{~W} / \mathrm{m}-\mathrm{K}$ is the thermal conductivity of a soot particle [4]. $C_{\mathrm{m}}=1.14$ and $C_{\mathrm{t}}=2.18$, moreover, are model parameters [11]. Toda et al. [66] conducted accurate measurements of thermophoresis in microgravity conditions and introduced a correction parameter $C_{\mathrm{s}}$ based on the diameter $d_{\mathrm{p}}$ of a spherical particle

$$
C_{\mathrm{s}}=2.72 \times 10^{5}\left(d_{\mathrm{p}}-2.7 \times 10^{-6}\right)+3.19 .
$$

For fractal aggregates, $d_{\mathrm{p}}$ in Eq. (4) is replaced by the diameter of gyration $d_{\mathrm{g}}$, see Section 3.4 .

The governing equations are discretised in $(\mathbf{x}, v)$ using a standard finite volume scheme [48] and incorporated into our in-house research software BOFFIN [32]. In order to allow for the application of tailored time integration schemes, the PBE in Eq. (1) is solved in two fractional steps [49, Section 6.3]. The first step involves convection and diffusion in physical space, using a Crank-Nicolson scheme, while the second fractional step encompasses convection and source/sink processes in particle volume space, employing the fifth order accurate Runge-Kutta scheme DOPRI5 [27]. For the cell face fluxes in $v$-space, we adopt the high resolution formulation of Koren [36], also see Qamar et al. [51].

The double integrals that occur in the aggregation source terms, moreover, are evaluated using the approach recently developed by Liu and Rigopoulos [45]. This approach is advantageous because of (a) its accurate prediction of the distribution for a small number of finite volume cells, (b) the conservation of the first moment (or any other single moment) during aggregation, (c) the applicability to an arbitrary non-uniform grid and (d) its speed and robustness when coupled with CFD. Liu and Rigopoulos [45] tested the approach on the original Santoro experiments [58] and found 60 finite volume cells in $v$-space to be sufficient for obtaining converged results. For this reason, 60 finite volume cells are also employed for the $v$-discretisation in the present work.

\subsection{Soot kinetics}

\subsubsection{Nucleation}

Frequently, the coalescence of two PAH molecules is considered as the main soot particle inception step [6, 9, 73]. Contrary to acetylene-based models, PAH dimerisation constitutes an intermediate stage between the gaseous phase and the mature solid phase. In the present article, we consider the dimerisation of PAHs with two or more aromatic rings as a newly formed soot nucleus. For the BBP and KM2 mechanisms, the dimerisation rate is computed by assuming a Maxwell velocity distribution in the kinetic theory of molecular collisions such that the nucleation rate (in $\left[1 / \mathrm{m}^{3}\right]$ ) is given by the collision frequency of two $\mathrm{PAH}$ molecules, $\mathrm{PAH}_{j}$ and $\mathrm{PAH}_{k}$,

$$
\dot{B}\left(\mathrm{PAH}_{j k}\right)=C_{\mathrm{r}} \gamma_{j k} N_{\mathrm{A}} d_{j k}^{2} \sqrt{\frac{8 \pi R_{\mathrm{J}} T}{M_{j k}}}\left[\mathrm{PAH}_{j}\right]\left[\mathrm{PAH}_{k}\right],
$$

where $N_{\mathrm{A}}$ denotes Avogadro's number, $R_{\mathrm{J}}$ is the gas constant (in $[\mathrm{kmol}-\mathrm{K} / \mathrm{kJ}]$ ) and $d_{j k}$ indicates the mean diameter of the molecules $\mathrm{PAH}_{j}$ and $\mathrm{PAH}_{k} . M_{j k}$, moreover, represents the equivalent molar mass of $\mathrm{PAH}_{j}$ and $\mathrm{PAH}_{k}$,

$$
M_{j k}=\frac{M_{j} M_{k}}{M_{j}+M_{k}},
$$

and square brackets indicate molar concentrations (in $\left[\mathrm{kmol} / \mathrm{m}^{3}\right]$ ). The PAH species and the associated dimerisation efficiencies $\gamma_{i}$ are chosen based on the original articles by Blanquart and Pitsch [9] and Wang et al. [73]. We maintain the custom that only the self collisions of a series of PAH molecules are considered in the BBP chemistry, whereas also heterogeneous collisions involving different PAHs is accounted for in the KM2 chemistry. The collision efficiencies $\gamma_{j k}$ for the heterogeneous dimerisation rates are computed as the geometric means of the associated single species collision efficiencies,

$$
\gamma_{j k}=\left\{\begin{array}{ll}
\gamma_{j} & \text { if } j=k \\
\sqrt{\gamma_{j} \gamma_{k}} & \text { otherwise }
\end{array} .\right.
$$

The correction constant $C_{\mathrm{r}}$ in Eq. [5] is set to 1.0 for the BBP mechanism [9] and to 0.127 for the KM2 mechanism [73]. As reference, Table 1 summarises the PAH molecules that are included in the dimerisation step for either reaction mechanism along with the molar masses $M_{i}$ and the single species collision efficiencies $\gamma_{i}$. This table also includes the PAH species and properties for the ABF mechanism. For consistency with the BBP chemistry and contrary to Appel et al. [6] who only included the dimerisation of pyrene, we base the ABF nucleation rate on the dimerisation of all those $\mathrm{PAHs}$ which the $\mathrm{ABF}$ and $\mathrm{BBP}$ mechanisms have in common. 


\begin{tabular}{lclccc}
\hline \multirow{2}{*}{ Species } & \multirow{2}{*}{ Molar mass $M_{i}[\mathrm{~kg} / \mathrm{kmol}]$} & \multicolumn{1}{c}{ Name } & \multicolumn{3}{c}{ Collision efficiency $\gamma_{i}$} \\
\cline { 4 - 6 } & & & ABF & BBP [9] & KM2 [73] \\
\hline $\mathrm{C}_{10} \mathrm{H}_{8}$ & 128.17 & Naphthalene & 0.0010 & 0.0010 & - \\
$\mathrm{C}_{12} \mathrm{H}_{8}$ & 152.20 & Acenaphthylene & 0.0030 & 0.0030 & - \\
$\mathrm{C}_{12} \mathrm{H}_{10}$ & 154.21 & Biphenyl & 0.0085 & 0.0085 & - \\
$\mathrm{C}_{14} \mathrm{H}_{10}$ & 178.24 & Phenathrene & 0.0150 & 0.0150 & - \\
$\mathrm{C}_{16} \mathrm{H}_{10}$ & 202.26 & Pyrene & 0.0250 & 0.0250 & 0.006 \\
& & Acephenanthrylene & - & 0.0250 & - \\
& & Fluoranthene & - & 0.0250 & - \\
$\mathrm{C}_{18} \mathrm{H}_{10}$ & 226.28 & Cyclo[cd]pyrene & 0.0390 & 0.0390 & - \\
& & 1-Ethynyl pyrene & - & - & 0.010 \\
& & 2-Ethynyl pyrene & - & - & 0.010 \\
$\mathrm{C}_{20} \mathrm{H}_{12}$ & 252.31 & 4-Ethynyl pyrene & - & - & 0.010 \\
$\mathrm{C}_{22} \mathrm{H}_{12}$ & & Benzo(a)pyrene & - & - & 0.011 \\
$\mathrm{C}_{24} \mathrm{H}_{12}$ & 276.34 & Benzo(e)pyrene & - & - & 0.011 \\
\hline
\end{tabular}

Table 1: List of PAH species that are involved in dimerisation and surface condensation for three chemical reaction mechanisms.

In the finite volume discretisation along $v$, the contribution of nucleation to the temporal rate of change of number density in the $i$ th finite volume cell $\left[v_{i-1}, v_{i}\right]$ with width $\Delta v_{i}=v_{i}-v_{i-1}$ is evaluated as

$$
\dot{B}_{i}=\sum_{j} \sum_{k \leq j} \frac{1}{\Delta v_{i}} \int_{v_{i-1}}^{v_{i}} \dot{B}\left(\mathrm{PAH}_{j k}\right) \delta\left(v-v\left(\mathrm{PAH}_{j k}\right)\right) \mathrm{d} v,
$$

where $v\left(\mathrm{PAH}_{j k}\right)$ is the volume of a dimer formed from $\mathrm{PAH}_{j}$ and $\mathrm{PAH}_{k}[23]$ and $\delta$ indicates Dirac's deltadistribution.

\subsubsection{Surface growth and condensation}

According to the HACA mechanism [6], surface reactions occur at specific sites that are distributed across the surface of a particle. These sites may either be associated with hydrogen atoms bonded to carbon atoms or radical sites on dehydrogenated carbon atoms. The number density of the first type of sites $\left(\mathrm{C}_{\text {soot }}-\mathrm{H}\right)$ is estimated constant at $2.3 \times 10^{15}$ sites $/ \mathrm{cm}^{2}$ based on the diameter of a benzene ring and the distance between PAH layers in soot [22]. The number density of radical sites $\left(\mathrm{C}_{\text {soot }} \cdot\right)$, on the other hand, is rationalised by assuming that both types of sites are in steady state. Besides the $\mathrm{H}$ and $\mathrm{OH}$ radicals, Hwang and Chung [31] and Wang et al. [73] emphasised that $\mathrm{CH}_{3}, \mathrm{C}_{3} \mathrm{H}_{3}$ and $\mathrm{C}_{2} \mathrm{H}$ also play an important role in hydrogen abstraction.

$$
\begin{array}{rll}
\mathrm{C}_{\text {soot }}-\mathrm{H}+\mathrm{CH}_{3} & \Leftrightarrow & \mathrm{C}_{\text {soot }} \cdot+\mathrm{CH}_{4} \\
\mathrm{C}_{\text {soot }}-\mathrm{H}+\mathrm{C}_{3} \mathrm{H}_{3} & \Leftrightarrow & \mathrm{C}_{\text {soot }} \cdot+\mathrm{C}_{3} \mathrm{H}_{4} \\
\mathrm{C}_{\text {soot }}-\mathrm{H}+\mathrm{C}_{2} \mathrm{H} & \Leftrightarrow & \mathrm{C}_{\text {soot }} \cdot+\mathrm{C}_{2} \mathrm{H}_{2}
\end{array}
$$

A complete list of the enhanced HACA mechanism including surface reaction rates can be found in Reference [73]. In order to model the experimentally observed dependence of reactive sites on particle size [28, 29] and temperature [14, 74], Frenklach and Wang [22] introduced the fraction $\alpha$ of active surface sites, which has been used to adjust the soot growth rate at high temperatures; Veshkini et al. [68] summarised values and submodels for $\alpha$. In this article, we consider $\alpha$ to be temperature dependent and adopt the functional form proposed by Guo et al. [26]. The coefficients in this representation are determined by manual adjustment in such a way that the experimental measurements of the line-of-sight integrated soot volume fraction are well reproduced in the ethylene flames, see Table 2

Particularly at low temperatures, soot surface growth is also enhanced by the condensation of PAHs onto the particle surface. Unlike the chemical surface reactions in the HACA mechanism, PAH condensation is the physical collision of a PAH molecule and a soot particle that is independent of active surface sites. In our model, all PAH 


\begin{tabular}{cllc}
\hline \multirow{2}{*}{ Reaction mechanism } & \multicolumn{2}{c}{$C_{\mathrm{r}}$} & \multicolumn{2}{c}{ Coefficients in $\alpha(T)=\beta_{u} \exp \left(T_{u} / T\right)$} \\
\cline { 3 - 4 } & & \multicolumn{1}{c}{$\beta_{u}$} & $T_{u}$ \\
\hline $\mathrm{ABF}$ & 1.0 & 0.0095 & 9000 \\
$\mathrm{BBP}$ & 1.0 & 0.0037 & 9000 \\
$\mathrm{KM} 2$ & 0.127 & 0.0025 & 9000 \\
\hline
\end{tabular}

Table 2: Correction constant $C_{\mathrm{r}}$ in the PAH-based nucleation and condensation rates (Eqs. (5) and (12)) and temperature dependency of the fraction of active surface sites $\alpha=\alpha(T)$ in the HACA mechanism for the three different chemical reaction mechanisms considered here. The coefficient $\beta_{u}$ was adjusted to approximately match the experimental profiles of the line-of-sight integrated soot volume fraction along the flame centrelines.

molecules which contribute to the nucleation step can condense onto the surface of a soot particle with the same collision efficiency $\gamma_{i}$ and correction factor $C_{\mathrm{r}}$ (Tables 1 and 2 . The rate of increase in soot mass per surface area (in $\left[\mathrm{kg} / \mathrm{m}^{2}-\mathrm{m}^{3}\right]$ ) due to PAH condensation is computed according to

$$
\dot{R}(\mathrm{PAH})=C_{\mathrm{r}} \sum_{i} \gamma_{i} \sqrt{\frac{R_{\mathrm{J}} T}{2 \pi M_{i}}}\left[\mathrm{PAH}_{i}\right] M c_{i},
$$

where $M c_{i}$ (in $[\mathrm{kg} / \mathrm{kmol}]$ ) denotes the carbon weight per mole for $\mathrm{PAH}_{i}$.

The cumulative volumetric growth rate encompasses contributions from the enhanced HACA mechanism for oxidation and acetylene-based growth as well as the PAH condensation process

$$
G(v, \mathbf{Y})=\frac{A_{\mathrm{s}}(v)}{\rho_{\mathrm{s}}}[\dot{R}(\mathrm{HACA})+\dot{R}(\mathrm{PAH})],
$$

where $\dot{R}(\mathrm{HACA})$ represents the surface-specific soot growth rate of the enhanced HACA mechanism and $\rho_{\mathrm{s}}=$ $1800 \mathrm{~kg} / \mathrm{m}^{3}$ is the density of solid soot. The formulae for the volume-dependent surface area $A_{\mathrm{s}}(v)$ of a soot particle are discussed in detail in Section 3.4 and summarised in Table 3 . If the growth rate $G(v, \mathbf{Y})$ in Eq. (13) is negative, then the oxidising species $\mathrm{O}_{2}$ and $\mathrm{OH}$ dominate the soot surface reactions.

\subsubsection{Particle aggregation}

The collision kernel in the aggregation term for the free molecular and continuum regimes, respectively, may be described according to [67]

$$
\begin{aligned}
\beta^{\mathrm{fm}}\left(v_{i}, v_{j}\right) & =2.2 \sqrt{\frac{\pi k_{\mathrm{B}} T}{\rho_{\mathrm{s}}\left(v_{i}+v_{j}\right)}}\left(d\left(v_{i}\right)+d\left(v_{j}\right)\right)^{2}, \\
\beta^{\mathrm{c}}\left(v_{i}, v_{j}\right) & =\frac{2 k_{\mathrm{B}} T}{3 \mu}\left(\frac{C\left(v_{i}\right)}{d\left(v_{i}\right)}+\frac{C\left(v_{j}\right)}{d\left(v_{j}\right)}\right)\left(d\left(v_{i}\right)+d\left(v_{j}\right)\right),
\end{aligned}
$$

where $k_{\mathrm{B}}$ denotes Boltzmann's constant, $d(v)$ represents either the diameter of a spherical particle $d_{\mathrm{p}}(v)$ or the diameter of gyration of an aggregate $d_{\mathrm{g}}(v)$ (Eqs. (18) and 21) below) and $C(v)$ is a function that encapsulates the Knudsen number dependency

$$
C(v)=1+1.257 \mathrm{Kn}(v) .
$$

The diameter of gyration $d_{\mathrm{g}}$ is a parameter used to measure the mass distribution in a fractal aggregate. We employ the free-molecular aggregation kernel $\beta^{\mathrm{fm}}$ for $\min \left(\operatorname{Kn}\left(v_{i}\right), \operatorname{Kn}\left(v_{j}\right)\right)>10$ and the continuum kernel $\beta^{\mathrm{c}}$ for $\max \left(\operatorname{Kn}\left(v_{i}\right), \operatorname{Kn}\left(v_{j}\right)\right)<0.1$. In-between these two conditions, the transition kernel $\beta^{\text {tr }}$ is used [50],

$$
\beta^{\operatorname{tr}}\left(v_{i}, v_{j}\right)=\frac{\beta^{\mathrm{fm}}\left(v_{i}, v_{j}\right) \beta^{\mathrm{c}}\left(v_{i}, v_{j}\right)}{\beta^{\mathrm{fm}}\left(v_{i}, v_{j}\right)+\beta^{\mathrm{c}}\left(v_{i}, v_{j}\right)} .
$$

\subsection{Soot morphology}

As previous research on soot morphology [65, 7, 44, 38] and our TEM observations indicate, soot particles tend to assemble into aggregates of fractal 'grape-like' shape, consisting of many connected spherical primary particles. 
In order to account for fractal shapes while maintaining a one-dimensional particle property space, we assume a soot particle to be spherical in shape if its volume is less than a critical value $v_{\mathrm{c} 1}$. On the other hand, it is assumed to be an aggregate consisting of several primary particles with identical volumes $v_{\mathrm{c} 1}$ if the volume of this aggregate is greater than another critical value $v_{\mathrm{c} 2}$. In-between these two limiting cases, soot particles may possess an intermediate shape whose morphological characteristics are detailed at the end of this section. Based on TEM images of thermophoretically sampled soot particles (Figure 3), we determined an average primary particle size of $d_{\mathrm{p}, \mathrm{a}}=30.8 \mathrm{~nm}$ which agrees well with the average size of $29 \mathrm{~nm}$ reported by Tian et al. [65]. For this reason, the first critical size is defined as the average size of primary particles $v_{\mathrm{c} 1}=v_{\mathrm{p}, \mathrm{a}}$; concomitantly, the second critical size is taken as $v_{\mathrm{c} 2}=10 v_{\mathrm{p}, \mathrm{a}}[10]$. The number of primary particles in a fractal-like aggregate can be estimated from the power law [24]

$$
N_{\mathrm{p}}(v)=\frac{v}{v_{\mathrm{p}, \mathrm{a}}}=k_{\mathrm{f}}\left(\frac{d_{\mathrm{g}}(v)}{d_{\mathrm{p}, \mathrm{a}}}\right)^{D_{\mathrm{f}}},
$$

where $v$ represents the volume of an aggregate, $d_{\mathrm{g}}(v)$ denotes the diameter of gyration, $k_{\mathrm{f}}=1.94$ is the fractal prefactor [44] and $D_{\mathrm{f}}=1.8$ is the fractal dimension [10]. For large non-spherical or elongated aggregates, the diameter of gyration and the hydrodynamic diameter are related according to [42]

$$
\frac{d_{\mathrm{g}}}{d_{\mathrm{h}}}=0.775
$$

Within an aggregate, neighbouring primary particles are assumed to be in point contact such that the surface area $A_{\mathrm{s}}(v)$ of an aggregate is estimated as [10]

$$
A_{\mathrm{s}}(v)=A_{\mathrm{p}, \mathrm{a}} N_{\mathrm{p}}(v)\left(1-\phi C_{\mathrm{ov}}\left(1-\frac{1}{N_{\mathrm{p}}(v)}\right)\right) .
$$

Here, $A_{\mathrm{p}, \mathrm{a}}$ represents the surface area of a spherical particle with diameter $d_{\mathrm{p}, \mathrm{a}}, C_{\mathrm{ov}}=0.15$ is an overlapping parameter for neighbouring primary particles and $\phi$ is defined as the coordination number (number of contacts per primary particles), approximated as 1.3 for large $N_{\mathrm{p}}(v)$.

The shadings of the particles imaged in Figure 3 indicate that soot particles are initially liquid-like and carbonise progressively, in agreement with the recent results of Kholghy et al. [35]. At heights above the burner of $20 \mathrm{~mm}$ in the non-smoking flame and $30 \mathrm{~mm}$ in the incipient-smoking flame, the liquid-like particles are generally larger than a primary particle and appear to possess a shape that is intermediate between a spherical particle and a fractal aggregate. Assuming that the volumes of these particles range in $\left[v_{\mathrm{c} 1}, v_{\mathrm{c} 2}\right]=\left[v_{\mathrm{p}, \mathrm{a}}, 10 v_{\mathrm{p}, \mathrm{a}}\right]$, we treat the associated morphological diameters $d_{\mathrm{h}}(v)$ and $d_{\mathrm{g}}(v)$ in a special way. For example, rather than using the statistically empirical expressions in Eqs. [18) and (19), the diameter of gyration and the hydrodynamic diameter in the size range $\left[v_{\mathrm{p}, \mathrm{a}}, 4 v_{\mathrm{p}, \mathrm{a}}\right]$ are computed based on the theoretical expressions for primary particles, dimers, trimers and tetramers derived by Sandkühler et al. [57] and fitted as cubic polynomials. For the size range [ $\left.4 v_{\mathrm{p}, \mathrm{a}}, 10 v_{\mathrm{p}, \mathrm{a}}\right]$, by contrast, linear interpolations based on the values at the two boundaries are used to maintain continuity over the whole size domain.

$$
\begin{aligned}
& d_{\mathrm{g}}(v)= \begin{cases}d_{\mathrm{p}, \mathrm{a}}\left[-0.1067 N_{\mathrm{p}}^{3}+0.6787 N_{\mathrm{p}}^{2}-1.0219 N_{\mathrm{p}}+1.45\right], & 1<N_{\mathrm{p}}<4 \\
d_{\mathrm{p}, \mathrm{a}}\left(0.1819 N_{\mathrm{p}}-4+0.6655\right), & 4<N_{\mathrm{p}}<10\end{cases} \\
& d_{\mathrm{h}}(v)= \begin{cases}d_{\mathrm{p}, \mathrm{a}}\left[-0.1701 N_{\mathrm{p}}^{3}+1.086 N_{\mathrm{p}}^{2}-1.6828 N_{\mathrm{p}}+1.77\right], & 1<N_{\mathrm{p}}<4 \\
d_{\mathrm{p}, \mathrm{a}}\left(0.2798 N_{\mathrm{p}}+0.41\right), & 4<N_{\mathrm{p}}<10\end{cases}
\end{aligned}
$$

In addition, the surface area of a particle in this intermediate size range is also fitted based on the two boundary values and the $2 / 3$ power of its volume, thus,

$$
A_{\mathrm{s}}(v)=A_{\mathrm{p}, \mathrm{a}}\left[1.72 N_{\mathrm{p}}(v)^{\frac{2}{3}}-0.72\right], \quad v_{\mathrm{p}, \mathrm{a}}<v<10 v_{\mathrm{p}, \mathrm{a}} .
$$

As reference, the morphological parameters of primary soot particles, aggregates and particles with an intermediate shape are summarised in Table 3

\footnotetext{
${ }^{1}$ Here, the diameter of gyration $d_{\mathrm{g}}$ and the hydrodynamic diameter $d_{\mathrm{h}}$ are distinguished. $d_{\mathrm{g}}$ plays an important role in the collisional kinetics of soot and is defined as the mass weighted average distance between the centre of a molecule and its mass elements. $d_{\mathrm{h}}$, by contrast, controls the spatial diffusion of particles and is defined as the diameter of a hard sphere that diffuses at the same rate as the molecule under consideration. In general, the values of $d_{\mathrm{g}}$ and $d_{\mathrm{h}}$ are of the same order of magnitude.
} 


\begin{tabular}{lllll}
\hline Geometrical parameter 1 & Symbol & $v \leq v_{\mathrm{p}, \mathrm{a}}$ & $v_{\mathrm{p}, \mathrm{a}}<v<10 v_{\mathrm{p}, \mathrm{a}}$ & $10 v_{\mathrm{p}, \mathrm{a}} \leq v$ \\
\hline Diameter of gyration & $d_{\mathrm{g}}$ & $(6 v / \pi)^{1 / 3}$ & Eq. (21) & $d_{\mathrm{p}, \mathrm{a}}\left(k_{\mathrm{f}} v / v_{\mathrm{p}, \mathrm{a}}\right)^{1 / D_{f}}$ \\
Hydrodynamic diameter & $d_{\mathrm{h}}$ & $d_{\mathrm{g}}$ & Eq. (22) & $d_{\mathrm{g}} / 0.775$ \\
Surface area & $A_{s}$ & $\pi d_{\mathrm{g}}^{2}$ & Eq. (23) & Eq. (20) \\
\hline
\end{tabular}

Table 3: Summary of the geometrical parameters we employ to describe the morphology of soot particles over a range of particle volumes $v$.

\section{Modelling of LIF, ELS and LOSA signals}

The central aspect of our model validation in Section 5] is to compare 'predicted laser diagnostic signals' computed from model predictions of major chemical species and the soot size distribution with laser diagnostic measurements, as advocated by Connelly et al. [13]. The intensity of the OH-PLIF signal, for instance, may be estimated according to [18]

$$
S^{\mathrm{OH}-\mathrm{PLIF}} \propto I_{\mathrm{inc}} n_{\mathrm{OH}} \sigma_{\mathrm{A}} Q_{\mathrm{Y}},
$$

where $I_{\mathrm{inc}}$ is the intensity of the incident laser beam with wavelength $\lambda$ and $n_{\mathrm{OH}}$ represents the number concentration of $\mathrm{OH}$ molecules. Additionally, $\sigma_{\mathrm{A}}$ and $Q_{\mathrm{Y}}$ denote the molecular absorption cross section and the quantum yield, respectively. Since both $\sigma_{\mathrm{A}}$ and $Q_{\mathrm{Y}}$ are functions of $\lambda$ and the local temperature $T$, their product is modelled in terms of the normalised Boltzmann fraction of electrons in the ground level $f_{v, J}(T)$ for a transition of quantum numbers $v, J$ at temperature $T$ and corrected by the collisional quenching of $\mathrm{OH}$ radicals by other major species $\left(\mathrm{N}_{2}, \mathrm{O}_{2}, \mathrm{H}_{2} \mathrm{O}, \mathrm{H}_{2}, \mathrm{CO}_{2}, \mathrm{CO}, \mathrm{H}\right.$ and $\left.\mathrm{OH}\right)$ using the quenching rates $w_{i}(T)$ of Tamura et al. [63]. Assuming that spontaneous emission is negligible compared with quenching, we hence calculate the normalised OH-PLIF signal as

$$
S^{\mathrm{OH}-\mathrm{PLIF}} \propto[\mathrm{OH}] \frac{f_{v, J}(T)}{w(T)} .
$$

For our purposes, the temperature dependence of the Boltzmann fraction is determined approximately with the aid of LIFBASE [3]

$$
f_{0,8}^{\mathrm{OH}}(T) \approx 1.206 \times 10^{-10} T^{3}-9.163 \times 10^{-7} T^{2}+2.146 \times 10^{-3} T-0.586
$$

and the local quenching rate is obtained as

$$
w(T)=\sum_{i} X_{i} w_{i}(T)
$$

where $X_{i}$ denotes the mole fraction of the colliding species $i$ and $w_{i}(T)$ is the corresponding quenching rate.

While the OH-PLIF signal $S^{\mathrm{OH}-\mathrm{PLIF}}$ is primarily proportional to the concentration of $\mathrm{OH}$, we emphasise that it also depends on the concentrations of other species (including $\mathrm{OH}$ ) via the quenching rate $w(T)$ and on temperature. Strictly, the dependency of $w(T)$ on $[\mathrm{OH}]$ renders $S^{\mathrm{OH}-\mathrm{PLIF}} \propto[\mathrm{OH}] g\left([\mathrm{OH}],\left[\mathrm{N}_{2}\right],\left[\mathrm{O}_{2}\right], \ldots, T\right)$, which is nonlinear and cannot be directly interpreted as a normalised $\mathrm{OH}$ concentration. Yet, even if the dependency of the quenching rate on $[\mathrm{OH}]$ were omitted, we would require estimates of temperature and other species involved in the quenching rate, for example, from an existing physical model, to infer the normalised $\mathrm{OH}$ concentration. By consequence, the $\mathrm{OH}$ concentration estimated from Eq. 25] is subject to a combination of modelling and experimental uncertainties. In order to circumvent this entanglement of physical modelling and measurement, we decided to directly compare the experimental OH-PLIF signal with its modelled counterpart $S^{\mathrm{OH}-\mathrm{PLIF}}$ in Section 5.2

The ELS signal intensity $S^{\text {ELS }}$ is modelled according to the Rayleigh-Debye-Gans theory of light scattering by fractal aggregates. Since we consider molecular light scattering to be negligible, $S^{\mathrm{ELS}}$ is proportional to both the number density and the scattering cross-section $\sigma_{\mathrm{s}}(v)$ of soot particles. The scattering cross-section may be computed according to [44]

$$
\sigma_{\mathrm{s}}(v)=k_{f} \begin{cases}k^{4} F(m)\left(\frac{d(v)}{2}\right)^{6} & \text { for primaries } \\ k^{4} F(m) S(q) N_{\mathrm{p}}(v)^{2}\left(\frac{d_{\mathrm{p}, \mathrm{a}}}{2}\right)^{6} & \text { for aggregates }\end{cases}
$$


where $k_{f}$ is the fractal prefactor, $k$ denotes the wavenumber of the incident light and $F(m)=\left|\left(m^{2}-1\right) /\left(m^{2}+1\right)\right|^{2}$ is a function of the complex refractive index of soot $(m=1.61-1.74 \mathrm{i}[76]) . S(q)$, moreover, represents a structure factor which adopts different expressions for the Rayleigh, Guinier and power-law scattering regimes [61] depending on the size parameter $q R_{\mathrm{g}}$

$$
S(q) \propto\left\{\begin{array}{ll}
1 & \text { if } q R_{g}<0.1 \\
1-\frac{\left(q R_{g}\right)^{2}}{3} & \text { if } 0.1 \leq q R_{g} \leq 1 \\
\left(q R_{g}\right)^{-D_{f}} & \text { otherwise }
\end{array} .\right.
$$

Here, $q=2 k \sin (\theta / 2)$ is the norm of the scattering wave vector, $\theta$ is the angle of observation and $R_{g}$ is half of the diameter of gyration. The relative ELS signal is thus calculated based on the soot particle size distribution according to

$$
S^{\mathrm{ELS}} \propto \int_{0}^{\infty} n(v, \mathbf{x}, t) \sigma_{\mathrm{s}}(v) \mathrm{d} v .
$$

Since our PBE-based model resolves the soot particle size distribution, the laser-diagnostic signals can be recovered without any assumption on the shape of the size distribution. This is important because it may not always be clear which shape is appropriate; monodispersed is most simple, log-normal is common [44] and a combination is sometimes employed [54].

The Beer-Lambert law describes how a laser beam is attenuated as it travels through a flame for a distance $L$ due to a combination of absorption and scattering by soot particles. Based on this law, the line-of-sight ISVF may be expressed in terms of the experimental LOSA signal $I / I_{0}$ according to

$$
\operatorname{ISVF}=\int_{0}^{L} f_{v}(s) \mathrm{d} s=-\frac{\lambda}{6 \pi E(m)} \ln \left(\frac{I}{I_{0}}\right),
$$

where $\lambda$ is the laser wavelength, $f_{v}(s)$ represents the soot volume fraction (spatial coordinate $\left.s \in[0, L]\right)$ and $E(m)$ denotes the absorption function

$$
E(m)=\operatorname{Im}\left(\frac{m^{2}-1}{m^{2}+1}\right) .
$$

Here, $\operatorname{Im}(\cdot)$ indicates the imaginary part of a complex number.

\section{Results and Discussion}

In the present section, model predictions are compared with experimental measurements on a signal-basis and characteristic traits in the spatial distribution of soot as well as their sensitity to changes in the gas phase and soot kinetics are elucidated. To assist interpretation, we briefly summarise the key differences in the soot models of the three mechanisms at this point. For both the ABF and BBP mechanisms, the nucleation and condensation rates are based on $\mathrm{A} 2$ to $\mathrm{A} 4$ and feature identical collision efficiencies (see Table 1) such that differences in the $\mathrm{ABF}$ and $\mathrm{BBP}$ nucleation/condensation rates are solely due to the gas phase chemistry. This is in contrast to the KM2 chemistry, which includes higher PAHs and for which the nucleation/condensation rates are based on the concentrations of A4 to A7. The coefficient $\beta_{u}$ in the HACA-based surface growth rate, moreover, is adjusted in Section 5.2 (see also Table 2) such that the predictions of the line-of-sight ISVF obtained for the three model formulations match, approximately, the counterpart deduced from the LOSA measurement by Eq. (31) (see Figure 5 below). This calibration was found to be necessary to level up the amount of soot formed in each of the three simulations and, thereby, establish a basis for assessing relative deviations in predicting soot formation. The kinetics for soot aggregation and oxidation are identical for all three reaction mechanisms.

\subsection{Gas phase chemistry in the absence of soot formation}

For the flow conditions of the incipient-smoking flame, Figure 4 depicts the radial profiles of key gas phase variables obtained with the ABF mechanism (based on GRI 1.2) and the BBP and KM2 mechanisms (both based on GRI 3.0) when soot formation is deactivated. As reference, we also include the corresponding profiles for the non-PAH-baseline mechanisms GRI 1.2 and GRI 3.0. While the ABF, BBP and KM2 mechanisms yield consistent predictions for the spatial distribution of temperature and $\mathrm{C}_{2} \mathrm{H}_{2}$ (apart from minor downstream deviations), the two non-PAH-based mechanisms (GRI 1.2 and GRI 3.0) differ noticeably. This indicates that the addition of PAH 
chemistry has a large impact on the overall reaction progress and the non-PAH composition. Since $\mathrm{C}_{2} \mathrm{H}_{2}$ is the main driver for HACA-based surface growth, soot growth may contribute similarly to increases in soot volume fraction in the three model formulations if the HACA-kinetics are kept consistent. On the other hand, Figure 4(b) indicates no consensus on the PAH profiles. Compared to the ABF chemistry, the BBP mechanism yields an almost order-of-magnitude increase in the $\mathrm{A}_{2}$ mole fraction as well as much wider $\mathrm{A}_{4}$ profiles, eliciting more vigorous nucleation and condensation. Indeed, in Section 5.2 consistency of the overall amount of soot in the ABF and BBP simulations is reestablished by decreasing the HACA growth coefficient $\beta_{u}$ for the BBP mechanism and, in this way, altering the relative contributions of nucleation/condensation and surface growth.

\subsection{Laser diagnostic signals}

In this section, we compare the measured OH-PLIF, ELS and LOSA signals with the corresponding model predictions. The line-of-sight ISVF is related to the LOSA signal according to Eq. 31. Figure 5 depicts both the measured and predicted line-of-sight ISVF along the centreline for the non-smoking and incipient-smoking flames. The solid red line represents the measured ISVF for a constant value of $E(m)=0.32$ based on a complex refractive index of $m=1.61-1.74 \mathrm{i}[76]$. The uncertainty in the ISVF is mainly due to the wide range of values reported for $E(m)$, see Appendix A Following Chang and Charalampopoulos [12] and Kelesidis and Pratsinis [34], $E(m)$ is mainly affected by the laser wavelength $\lambda$, but may also be influenced by the type of fuel and soot maturity. Tian et al. [64] reported values of the absorption function $E(m)$ in the range from 0.21 to 0.37 for $\lambda \approx 632 \mathrm{~nm}$, while Kelesidis and Pratsinis [34] mentioned that, for nascent soot, $E(m)$ may be as low as 0.17 and increase as the soot particles mature. For this reason, we assume $0.17 \leq E(m) \leq 0.37$, corresponding to an uncertainty in the ISVF of over $50 \%$ as indicated by the shaded area in Figure 5 Since soot precursors and small soot particles attenuate much less laser energy than mature particles, the experimental ISVF computed from the LOSA signal may be underestimated by taking $E(m)=0.32$. Correspondingly, the dashed red line in Figure 5 indicates a guessed ISVF profile obtained using a minimum $E(m)$-value for small particles and a maximum value for mature aggregates.

In order to level the predictions of the amount of soot formed for the three reaction mechanisms, we adjusted the coefficient $\beta_{u}$ in the HACA surface growth model manually and approximately matched the predicted ISVF profiles with the experimental one for $E(m)=0.32$. Despite this one-parameter tuning, the ISVF predictions continue to show qualitative deviations. For example, the ISVF peak is underpredicted and the modelled ISVF decays about $5 \mathrm{~mm}$ to $15 \mathrm{~mm}$ further downstream than the experimental profile. If the $\beta_{u}$-values in Table 2 are further increased or decreased, then either of these discrepancies is aggravated. A reduction in $\beta_{u}$, for instance, results in an upstream shift of the decaying branch of the profile, but also causes the maximum ISVF value to decrease significantly. If the experimental ISVF is corrected for the change of $E(m)$ with size (dashed red line in Figure 5), then the qualitative discrepancies in the predicted ISVF profiles appear to be slightly less severe, corroborating the impact of soot polydispersity on $E(m)$.

Complementary to Figure 5 , Figure 6 depicts the predicted and measured radial profiles of the line-of-sight ISVF for both flames. As above, the LOSA measurements are endowed with the uncertainty range detailed in Appendix A. In line with our previous observations, the radial ISVF peaks at the axial distances of $30 \mathrm{~mm}$ and $50 \mathrm{~mm}$ for the non-smoking and incipient-smoking flames, respectively, are lower than the values computed from the experimental LOSA signal (for $E(m)=0.32$ ). The experimental ISVF profiles indicate two sooty flame wings which are also present in the signals predicted by the ABF-based model. Quantitatively, however, the distance between the two sooty wings is overpredicted by about $2 \mathrm{~mm}$ in the ABF simulations. For the BBP and KM2 reaction mechanisms, by contrast, the radial increase in the line-of-sight ISVF away from the centreline and the subsequent drop are less pronounced and the radial profiles appear to be much smoother.

The left panels in Figure 7 show the radial profiles of the measured and predicted normalised ELS signal at several downstream positions for the two flames. The radial locations at which the ELS signal peaks are correctly reproduced by the BBP and KM2 mechanisms, while the maxima are displaced radially outwards for the $\mathrm{ABF}$ signals, echoing our observation in Figure 6. Additionally, the experimental ELS signal indicates that a significant amount of light is scattered close to the centreline in-between the sooty flame wings. This is also reflected in the BBP and KM2 predictions, but seems to be absent in the ABF simulation. For a different reaction mechanism, Dworkin et al. [16] and Eaves et al. [17] obtained predictions of the centreline soot volume fraction in the same order of magnitude as the corresponding measurements. They argued that PAH-based nucleation dominates on the centreline and found their reaction mechanism to lead to much larger centreline PAH concentrations than the ABF mechanism. The focus on PAH chemistry is also evidenced by previous observations on the underprediction of the soot volume fraction near the centreline for purely acetylene-based nucleation models [46, 5, 25].

The right panels in Figure 7 depict measured and predicted radial profiles of the OH-PLIF signal. Throughout 
the flames, the radial peaks of the predicted normalised OH-PLIF signals are slightly narrower than the experimental observations. As the axial distance increases, the OH-PLIF peaks migrate towards the centreline until they partially overlap with the sooty flame wings. For both flames, the measured OH-PLIF profiles are slightly more skewed towards the centreline at the downstream distances than the predicted profiles and $\mathrm{OH}$ is also detected near the flame centre. Physically, soot forms and grows on the rich side of the flame and is oxidised, mainly by $\mathrm{OH}$, in the vicinity of stoichiometric conditions. This rationale is corroborated by the observation that the predicted ELS and OH-PLIF profiles in Figure 7 are mainly non-overlapping, except by a small amount at the furthest downstream location.

Further to Figure 7, Figures 8 and 9 show contour plots of the predicted and measured normalised ELS and OH-PLIF signals in the non-smoking and incipient-smoking flames. The white gaps in the experimental ELS contour plot indicate regions for which ELS data was not obtained. OH-PLIF data, by contrast, was collected in a series of overlapping stripes as a function of height. The rightmost panels in Figures 8 and 9 therefore provide an honest illustration of experimental reproducibility; some minor discontinuities can be observed at the 'seam' between stripes, caused by residual flame 'meandering' despite the presence of a shrouding chimney. The experimental records show that the two sooty wings appear approximately $15 \mathrm{~mm}$ above the nozzle exit plane and disappear at axial distances of about $60 \mathrm{~mm}$ and $95 \mathrm{~mm}$, respectively. Although the sooty wings are also prominent in the predicted ELS signals, the three different gas phase mechanisms lead to qualitative differences. For the BBP and KM2 mechanisms, for instance, soot formation seems to commence further upstream than in the experimental records and soot persists until the wings converge on the centreline near the flame tip. Additionally, the sooty wings are thicker, eliciting a strong ELS signal from the centreline upstream of the flame tip. This is quite in contrast to the ELS contours obtained from the ABF mechanism. Here, the sooty wings are almost as sharp as indicated by the experimental measurements; however, as in Figure 7, the wings are shifted radially outwards and there does not seem to be any light scattering near the centreline. The increase in thickness of the sooty wings in the BBP and KM2 predictions compared to the experimental ELS signal may be due to differences in the sensitivity towards the presence of soot nuclei. In our PBE-based model, PAH dimers appear as soot nuclei and are accounted for in the soot particle size distribution and the predicted ELS signal. These dimers are, however, too small to bear an influence on the measured ELS signal or to be sampled intrusively. By consequence, the experimental ELS signal and the soot size statistics obtained from the TEM images are biased towards larger particle sizes. At the same time, we surmise that the sooty wings in the ABF predictions inherit their sharpness from the upstream profiles of the nucleation rate (Figure $10(\mathrm{c})$ below).

Apart from the downstream tails, the experimental OH-PLIF signals almost completely envelop the ELS signals. The experimental measurements indicate that the OH-PLIF wings emanate from the edges of the fuel tube and convene on the centreline about $70 \mathrm{~mm}$ and $100 \mathrm{~mm}$ above the burner exit plane, respectively. By contrast, the downstream tip of the predicted OH-PLIF contours is located approximately $10 \mathrm{~mm}$ to $15 \mathrm{~mm}$ further downstream than in the experimental records, echoing the delay in soot oxidation of Figures 5 to 9

In summary, some discrepancies persist between our model predictions and experimental laser diagnostic signals despite the calibration to the experimental ISVF profile on the centreline. In terms of the location and intensity of soot production, the PBE-based soot model relies on the outcome of the reaction mechanism used to describe the gas phase chemistry and pool of soot precursors. In particular, the predictions obtained from the BBP and KM2 mechanisms reproduce the distance between the sooty flame wings very well. On the centreline, the experimental measurements indicate that a small amount of soot is produced, but this is overestimated by the BBP and KM2 mechanisms and completely absent in the predictions obtained with the ABF chemistry.

\subsection{Radial profiles of key variables controlling soot formation}

In the present section, we turn to the analysis of the spatial distribution of key gas phase variables along with the nucleation, surface growth, condensation and oxidation rates they control. For brevity, model predictions are shown only for the incipient-smoking flame, but we emphasise that the corresponding predictions for the non-smoking flame share similar features.

Figure 10(a) depicts radial profiles of temperature and of the $\mathrm{C}_{2} \mathrm{H}_{2}$ mole fraction at different heights above the burner exit in the presence of soot formation. Beyond an axial distance of about $30 \mathrm{~mm}$, the temperature profiles obtained from the ABF mechanism fall below the BBP and KM2 profiles by up to $200 \mathrm{~K}$, although the three mechanisms yielded coincident temperature profiles in the simulations without modelling of soot formation (Figure 4(a). This seems to indicate that the ABF results are much heavier influenced by soot radiation and species scavenging than the BBP and KM2 predictions. Since the line-of-sight ISVF along the centerline has been approximately matched for the three mechanisms, but the sooty wings are located further away from the centreline 


\begin{tabular}{llll}
\hline \multicolumn{1}{c}{ Case } & Abbreviation & $C_{\mathrm{r}}$ & $\alpha$ \\
\hline Original & OR & 1.0 & $0.0037 \times \exp (9000 / T)$ \\
Adjusted 1 & AD1 & 0.1 & $0.0062 \times \exp (9000 / T)$ \\
Adjusted 2 & AD2 & 0.01 & $0.0105 \times \exp (9000 / T) \approx 1.0$ \\
\hline
\end{tabular}

Table 4: Summary of the parameter sets $\left(C_{\mathrm{r}}, \alpha\right)$ considered for a sensitivity analysis with respect to the rates of nucleation and surface growth.

for the $\mathrm{ABF}$ mechanism than for $\mathrm{BBP}$ or $\mathrm{KM} 2$, the flame area and the cross-sectional ISVF are larger in the $\mathrm{ABF}$ case, eliciting intenser radiative heat losses.

The $\mathrm{C}_{2} \mathrm{H}_{2}$ profiles obtained from the three reaction mechanisms, by contrast, match for a larger axial range, up until about $50 \mathrm{~mm}$ above the burner exit. Further downstream, the $\mathrm{C}_{2} \mathrm{H}_{2}$ profiles obtained from the $\mathrm{ABF}$ mechanism extend slightly further away from the centreline than the BBP and KM2 profiles, reflecting the radial shift in the sooty wings we observed with the ABF predictions in Figures 6(b), 7(b) and 9(a). By an axial distance of $90 \mathrm{~mm}, \mathrm{C}_{2} \mathrm{H}_{2}$ has completely disappeared for the ABF and BBP mechanisms, whereas the KM2 profiles still indicate a small amount of $\mathrm{C}_{2} \mathrm{H}_{2}$ here. This may explain why the ELS signals predicted by the KM2 mechanism vanish further downstream (Figure 9(a)). Interestingly, the $\mathrm{C}_{2} \mathrm{H}_{2}$ profiles extend further downstream than in the predictions obtained when soot formation is deactivated (Figure 4(a), , potentially because the temperature drop due to soot radiation has a retarding effect on the hydrocarbon reactions.

Since A2 serves as the main species for PAH dimerisation and condensation in the ABF and BBP mechanisms, Figure 10(b) shows profiles of the A2 mole fraction along with A4. In the early to middle stages of soot formation (from $10 \mathrm{~mm}$ to $50 \mathrm{~mm}$ above the burner exit), the A2 mole fraction predicted by the BBP mechanism is about one to two orders of magnitude larger than the one obtained from the ABF mechanism. The A4 mole fraction, on the other hand, is orders of magnitude lower than the A2 mole fraction in both the ABF and BBP simulations. This is at odds with the KM2 results, where the A4 mole fraction takes on similar values as the A2 mole fraction, supporting the selection of higher PAHs (A4 to A7) as soot precursors in the KM2 nucleation process following Wang et al. [73]. Physically, these large PAHs are thought to play an important role in soot nucleation [23, 35].

Figure 10(c), moreover, depicts radial profiles of the nucleation and growth rates in the incipient-smoking flame. Here, the profiles of nucleation rate correspond well with the spatial distribution of the PAHs in Figure $10(\mathrm{~b})$, while the growth rate peaks in regions of high temperature and large $\mathrm{C}_{2} \mathrm{H}_{2}$ concentration. Despite the same nucleation model, the nucleation rates computed from the $\mathrm{ABF}$ and $\mathrm{BBP}$ mechanisms differ by one to two orders of magnitude (in line with differences in the corresponding PAH profiles in Figure $10(\mathrm{~b})$, leading to significantly different spatial distributions of soot. This is at variance with the BBP and KM2 simulations, which are characterized by similar nucleation rates and lead to similar spatial soot distributions, although the respective nucleation models differ. Commensurate with the adjustment of the parameter $\beta_{u}$ in the HACA model (Table 2), the maximum surface growth rates at $15 \mathrm{~mm}$ and $35 \mathrm{~mm}$ above the burner exit slightly decrease from the ABF to the BBP and KM2 mechanisms despite the similarities in the $\mathrm{C}_{2} \mathrm{H}_{2}$ profiles.

Finally, Figure 10(d) shows the specific surface oxidation rate due to $\mathrm{OH}$ and $\mathrm{O}_{2}$ alongside the PAH surface condensation rate. The oxidation profiles coincide for the three different reaction mechanisms almost up to the flame tip, indicating that the spatial distributions of $\mathrm{OH}$ and $\mathrm{O}_{2}$ are only slightly affected by a change in gas phase chemistry. The condensation rate is shown on the same scale as the surface growth rate and we observe that PAH addition occurs predominantly near the centreline, while $\mathrm{C}_{2} \mathrm{H}_{2}$ addition is stronger in the sooty flame wings.

\subsection{Sensitivity analysis on nucleation and growth rates}

In the present section, we explore the sensitivity of the predicted line-of-sight ISVF and ELS signals with respect to the nucleation and growth rates. In Eqs. (5) and (12), the dimerisation and condensation rates are scaled by a correction factor $C_{\mathrm{r}}$. Similarly, the HACA surface growth rate is parameterised by the fraction of active sites $\alpha$. In order to maintain the experimental levels of the line-of-sight ISVF, the changes in $C_{\mathrm{r}}$ and $\alpha$ are coordinated as indicated in Table 4. As base test case, we consider the incipient-smoking flame using the BBP reaction mechanism because of the broad PAH profiles it yielded (Figure 10(b)) and otherwise reasonable predictions.

The $\left(C_{\mathrm{r}}, \alpha\right)$ parameter sets in Table 4 indicate that soot formation is much less sensitive to the nucleation and condensation rates than the growth rate. For example, a ten-fold reduction of the nucleation/condensation rate is accompanied by a less than two-fold increase in the growth rate if the axial profile of the experimental line-ofsight ISVF should be maintained. As the relative contributions of nucleation/condensation and growth change, 
the qualitative shape of the predicted profile remains almost intact (Figure 11). One slight improvement occurs in the nearfield (height $<20 \mathrm{~mm}$ ) where the reduction of the nucleation rate causes the line-of-sight ISVF on the centreline to decrease. However, the main limitations which we identified in Section 5.2 remain; the maximum line-of-sight ISVF is severely underpredicted and the downstream decay is delayed.

Figure 12 shows the influence of the $\left(C_{\mathrm{r}}, \alpha\right)$-set on the radial profiles of key variables which control soot formation. Although the line-of-sight ISVF remains very similar to the original profile for the first adjusted case, the corresponding spatial distribution of the ELS signal changes noticeably. Up to an axial distance of $35 \mathrm{~mm}$, the ELS intensity is almost halved, echoing the slight backward shift of the line-of-sight ISVF profile in Figure 11. Concomitantly, the nucleation rate decreases by less than an order of magnitude and the ten-fold reduction of $C_{\mathrm{r}}$ seems to be counterbalanced by the increase in PAH mole fractions caused by reduced species scavenging. The increase in the surface growth rate, on the other hand, takes effect about 2.5 to $5 \mathrm{~mm}$ away from the centreline and causes the sooty flame wings to shift radially outwards by a small amount.

As the nucleation and condensation rates are further reduced to a fraction of $C_{\mathrm{r}}=0.01$, we find that the predicted ELS profiles are qualitatively more similar to those obtained from the ABF mechanism than the BBP base case. In particular, the sooty wings are displaced in the radial direction. However, as a positive side effect and contrary to the ABF results, some soot remains in-between the wings on the centreline and the ELS signal emitted here appears to be quantitatively more accurate than the base BBP results. In summary, we observe that low levels of PAHs, small PAH-dimerisation and condensation rates and a strong surface growth rate result in a radial displacement of the sooty wings for ethylene diffusion flames along with a reduction in soot formation near the centreline. This finding is commensurate with the physical picture that the centreline soot mainly originates from PAH-based nucleation and condensation [16].

\section{Conclusions}

In this article, we presented a combined experimental and modelling investigation whose objective was to study the gas phase chemistry and soot formation in a laminar diffusion flame and to provide guidance on kinetic model adjustments. Planar OH laser-induced fluorescence (OH-PLIF), elastic light scattering (ELS) and line-of-sight attenuation (LOSA) measurements were used to characterise the flame structure and spatial distribution of soot and to quantify the line-of-sight integrated soot volume fraction (ISVF). The experimental dataset was supplemented by location-specific TEM images of soot particles extracted with the aid of a thermophoretic sampling device.

Our physical model integrates the population balance equation governing the soot particle size distribution into a reactive flow solver for multicomponent ideal gases. Besides detailed gas phase chemical kinetics, the model incorporates a complete set of PAH-based nucleation, condensation, HACA-based surface growth and oxidation kinetics as well as size-dependent aggregation. Based on morphological considerations, the surface areas and geometrical properties of soot particles were estimated separately for primary particles and aggregates. A particular feature of the simulation framework is a recently developed method for solving the population balance equation that combines accuracy in the prediction of the distribution with conservation of the soot volume fraction during aggregation [45].

Following the methodology advocated by Connelly et al. [13], the experimental measurements were compared with modelled signals based on predictions of the local gas phase composition and soot particle size distribution. On part of the OH-PLIF signal, this had the advantage that the experimental data could be kept distinct from the physical model; for example, temperature and the concentrations of species other than $\mathrm{OH}$ are commonly estimated when inferring the $\mathrm{OH}$ concentration from the OH-PLIF signal. Furthermore, the LOSA signal was converted to a line-of-sight ISVF instead of a local or cross-sectional ISVF, thus avoiding additional errors owing to tomographic inversions. Finally, the ELS signal was predicted by our PBE-based model, rather than being converted into a measure of mean particle size by assuming a particular shape of the size distribution.

Three well-established PAH-based chemical reaction mechanisms (ABF, BBP and KM2) were employed to model the inception of soot precursors and oxidants. An empirical parameter in the HACA mechanism, the fraction of active sites, had to be adjusted to qualitatively level up the model predictions for the three reaction mechanisms and, approximately, match the experimental line-of-sight ISVF profile on the centreline. This calibration reflects the limitations of present PAH kinetics and soot formation models. Despite the calibration, the comparison of the predicted and measured laser-diagnostic signals revealed several qualitative discrepancies. In particular, the maximum amount of soot was underpredicted and the downstream decay of soot was delayed. For the BBP and KM2 mechanisms, the radial distance between the sooty flame wings was accurately reproduced, while the centreline soot was slightly overestimated. Incorporation of the ABF mechanism, by contrast, led to a radial outward shift of the flame wings and a disappearance of soot near the centreline. This was, possibly, caused by the difference in 
gas phase chemistry and mediated by the PAH pool fuelling nucleation and condensation. Our sensitivity analyses indicated that the spatial distribution of soot is mainly controlled by the balance between nucleation/condensation and HACA-based surface growth. For example, a reduction of the nucleation/condensation rate and a coordinated increase in the surface growth rate cause a depletion of precursors and soot in the nearfield along with a radial outward shift of the sooty flame wings.

\section{Acknowledgements}

Anxiong Liu gratefully acknowledges financial support by the China Scholarship Council (CSC). The authors thank Prof. Richard Stone (Department of Engineering Science, University of Oxford) for the loan of the burner.

\section{Appendix A Uncertainty analysis of the LOSA measurements and line- of-sight ISVF evaluation}

The uncertainty in the line-of-sight ISVF computed from the LOSA signal by Eq. 31 comprises of two sources. The first one encompasses random errors that are attributed to the LOSA measurement system, including instrumentation precision and flame meandering. The second one is a systematic error incurred by the value of the absorption function $E(m)$ in Eq. (31). The instrumentation error originates from the intrinsic uncertainty in the measurement of light intensity. The instrumentation precision is $\pm 0.05 \mathrm{mV}$ relative to a signal of $22.3 \mathrm{mV}$, leading to a $\pm 0.24 \%$ error in the determination of the extinction coefficient. Since flame fluctuations affect the local soot volume fraction, the signal was averaged over a time period of $1 \mathrm{~s}$ for each measurement, resulting in a signal stability that is better than $\pm 0.05 \mathrm{mV}$.

The energy absorption $E(m)$ is a function of the complex refractive index $m$ of soot. Tian et al. [64] collated values of $m$ and $E(m)$ from previous investigations on smoking flames with various laser wavelengths and fuels. For a wavelength of about $632 \mathrm{~nm}$, the values of $E(m)$ are reported to range from 0.17 to 0.37 , whence we adopted, for a baseline calculation of the line-of-sight ISVF, $E(m)=0.32$.

By applying an analytical error propagation technique to Eq. 31, the mean square error $\Delta \mathrm{ISVF}^{2}$ in the line-of-sight ISVF computed from the LOSA signal can be estimated according to

$$
\Delta \mathrm{ISVF}^{2}=\left[\frac{\lambda}{6 \pi E(m)^{2}} \ln \left(\frac{I}{I_{0}}\right) \Delta E(m)\right]^{2}+\left(\frac{\lambda}{6 \pi E(m)}\right)^{2}\left[\left(\frac{\Delta I}{I}\right)^{2}+\left(\frac{\Delta I_{0}}{I_{0}}\right)^{2}\right] .
$$

Based on Eq. 33, the relative uncertainty reads

$$
\left(\frac{\Delta \mathrm{ISVF}}{\mathrm{ISVF}}\right)^{2}=\left[-\frac{\Delta E(m)}{E(m)}\right]^{2}+\left[\ln \left(\frac{I}{I_{0}}\right)\right]^{-2}\left[\left(\frac{\Delta I}{I}\right)^{2}+\left(\frac{\Delta I_{0}}{I_{0}}\right)^{2}\right] .
$$

If the error in the incident laser signal $\Delta I_{0}$ is taken as $0.05 \mathrm{mV}$ following our argument above for a nominal $I_{0}$ of $22.3 \mathrm{mV}$, then the relative error $\Delta I_{0} / I_{0}$ amounts to $\lesssim 2.2 \times 10^{-3}$. Since the strength of the received laser signal varies depending on the absorption intensity by soot, the ratio $\Delta I / I$ is generally larger than $\Delta I_{0} / I_{0}$ for a similar systematic error of $\Delta I_{0}=0.05 \mathrm{mV}$. The value for $-\Delta E(\mathrm{~m}) / E(\mathrm{~m})$, by contrast, ranges from -0.16 to 0.50 and constitutes the main bias in $\triangle \mathrm{ISVF} / \mathrm{ISVF}$. The largest relative error of 0.5 is indicated by the shaded areas in Figures 5,6 and 11.

An alternative approach to postprocess the LOSA signal consists in a tomographic inversion based on the Abel transform, resulting in an estimate of the local soot volume fraction. Since this introduces an additional algorithmic error, we restricted the consideration to the line-of-sight ISVF.

\section{References}

[1] (2003). http://www.sandia.gov/tnf/radiation.html.

[2] (2008). Theory Manual: Chemkin-Pro for Reaction Design. San Diego.

[3] (2016). https://www.sri.com/engage/products-solutions/lifbase. 
[4] Akbar, M. K. and Ghiaasiaan, S. M. (2005). Radiation heat transfer and soot thermophoresis in laminar tube flow. Numerical Heat Transfer, Part A: Applications, 47(7):653-670.

[5] Akridis, P. and Rigopoulos, S. (2017). Modelling of soot formation in laminar diffusion flames using a comprehensive CFD-PBE model with detailed gas-phase chemistry. Combustion Theory and Modelling, 21(1):35-48.

[6] Appel, J., Bockhorn, H., and Frenklach, M. (2000). Kinetic modeling of soot formation with detailed chemistry and physics: Laminar premixed flames of $\mathrm{C}_{2}$ hydrocarbons. Combustion and Flame, 121(1):122-136.

[7] Bladh, H., Johnsson, J., and Bengtsson, P.-E. (2009). Influence of spatial laser energy distribution on evaluated soot particle sizes using two-colour laser-induced incandescence in a flat premixed ethylene/air flame. Applied Physics B, 96(4):645-656.

[8] Blanquart, G., Pepiot-Desjardins, P., and Pitsch, H. (2009). Chemical mechanism for high temperature combustion of engine relevant fuels with emphasis on soot precursors. Combustion and Flame, 156(3):588-607.

[9] Blanquart, G. and Pitsch, H. (2009). A joint volume-surface-hydrogen multi-variate model for soot formation. In Bockhorn, H., D’ Anna, A., Sarofim, A. F., and Wang, H., editors, Combustion Generated Fine Carbonaceous Particles, pages 437-463. KIT Scientific Publishing, Karlsruhe.

[10] Brasil, A. M., Farias, T. L., and Carvalho, M. G. (1999). A recipe for image characterization of fractal-like aggregates. Journal of Aerosol Science, 30(10):1379-1389.

[11] Brock, J. R. (1962). On the theory of thermal forces acting on aerosol particles. Journal of Colloid Science, 17(8):768-780.

[12] Chang, H.-c. and Charalampopoulos, T. T. (1990). Determination of the wavelength dependence of refractive indices of flame soot. Proceedings of the Royal Society of London. Series A: Mathematical and Physical Sciences, 430(1880):577-591.

[13] Connelly, B. C., Bennett, B. A. V., Smooke, M. D., and Long, M. B. (2009). A paradigm shift in the interaction of experiments and computations in combustion research. Proceedings of the Combustion Institute, 32(1):879-886.

[14] Dasch, C. J. (1985). The decay of soot surface growth reactivity and its importance in total soot formation. Combustion and Flame, 61(3):219-225.

[15] Dobbins, R. A. and Megaridis, C. M. (1987). Morphology of flame-generated soot as determined by thermophoretic sampling. Langmuir, 3(2):254-259.

[16] Dworkin, S. B., Zhang, Q., Thomson, M. J., Slavinskaya, N. A., and Riedel, U. (2011). Application of an enhanced PAH growth model to soot formation in a laminar coflow ethylene/air diffusion flame. Combustion and Flame, 158(9):1682-1695.

[17] Eaves, N. A., Zhang, Q., Liu, F., Guo, H., Dworkin, S. B., and Thomson, M. J. (2016). CoFlame: A refined and validated numerical algorithm for modeling sooting laminar coflow diffusion flames. Computer Physics Communications, 207:464-477.

[18] Einecke, S., Schulz, C., and Sick, V. (2000). Measurement of temperature, fuel concentration and equivalence ratio fields using tracer LIF in IC engine combustion. Applied Physics B, 71(5):717-723.

[19] Eiteneer, B. and Frenklach, M. (2003). Experimental and modeling study of shock-tube oxidation of acetylene. International Journal of Chemical Kinetics, 35(9):391-414.

[20] El-Leathy, A. M., Kim, C. H., Faeth, G. M., and Xu, F. (2004). Soot surface reactions in high-temperature laminar diffusion flames. AIAA Journal, 42(5):988-996.

[21] Fairweather, M., Jones, W. P., Ledin, H. S., and Lindstedt, R. P. (1992). Predictions of soot formation in turbulent, non-premixed propane flames. Symposium (International) on Combustion, 24(1):1067-1074.

[22] Frenklach, M. and Wang, H. (1991). Detailed modeling of soot particle nucleation and growth. Symposium (International) on Combustion, 23(1):1559-1566. 
[23] Frenklach, M. and Wang, H. (1994). Detailed mechanism and modeling of soot particle formation. In Bockhorn, H., editor, Soot formation in combustion: Mechanisms and models, pages 165-192. Springer-Verlag, Berlin, Heidelberg.

[24] Friedlander, S. K. (2000). Smoke, Dust, and Haze, volume 198. Oxford University Press, New York.

[25] Garcia-Gonzalez, C. E., Sewerin, F., Liu, A., Rigopoulos, S., and Williams, B. A. O. (2017). Modelling of soot formation in a laminar coflow non-premixed flame with a detailed CFD-population balance model. In European Combustion Meeting 2017, Dubrovnik, Croatia.

[26] Guo, H., Liu, F., Smallwood, G. J., and Gülder, Ö. L. (2006). Numerical study on the influence of hydrogen addition on soot formation in a laminar ethylene-air diffusion flame. Combustion and Flame, 145(1):324-338.

[27] Hairer, E., Nørsett, S. P., and Wanner, G. (1993). Solving Ordinary Differential Equations I: Nonstiff Problems, volume 8 of Springer Series in Computational Mathematics. Springer-Verlag, Berlin, Heidelberg.

[28] Harris, S. J. and Weiner, A. M. (1985). Chemical kinetics of soot particle growth. Annual Review of Physical Chemistry, 36(1):31-52.

[29] Haynes, B. S. and Wagner, H. G. (1981). Soot formation. Progress in Energy and Combustion Science, 7(4):229-273.

[30] Hulburt, H. M. and Katz, S. (1964). Some problems in particle technology: A statistical mechanical formulation. Chemical Engineering Science, 19(8):555-574.

[31] Hwang, J. Y. and Chung, S. H. (2001). Growth of soot particles in counterflow diffusion flames of ethylene. Combustion and Flame, 125(1):752-762.

[32] Jones, W. P., di Mare, F., and Marquis, A. J. (2002). LES-BOFFIN: User's Guide. Imperial College London, Department of Mechanical Engineering, London.

[33] Kazakov, A., Wang, H., and Frenklach, M. (1995). Detailed modeling of soot formation in laminar premixed ethylene flames at a pressure of 10 bar. Combustion and Flame, 100(1):111-120.

[34] Kelesidis, G. A. and Pratsinis, S. E. (2019). Soot light absorption and refractive index during agglomeration and surface growth. Proceedings of the Combustion Institute, 37(1):1177-1184.

[35] Kholghy, M. R., Veshkini, A., and Thomson, M. J. (2016). The core-shell internal nanostructure of soot - A criterion to model soot maturity. Carbon, 100:508-536.

[36] Koren, B. (1993). A robust upwind discretization method for advection, diffusion and source terms. In Vreugdenhil, C. B. and Koren, B., editors, Numerical Methods for Advection-Diffusion Problems, volume 45 of Notes on Numerical Fluid Mechanics and Multidisciplinary Design, pages 117-138. Vieweg Verlag.

[37] Kosfeld, R. (1998). The physics of polymers, concepts for understanding their structures and behavior. Zeitschrift für Physikalische Chemie, 206(1-2):274-276.

[38] Köylü, Ü. Ö., Xing, Y., and Rosner, D. E. (1995). Fractal morphology analysis of combustion-generated aggregates using angular light scattering and electron microscope images. Langmuir, 11(12):4848-4854.

[39] Kreyling, W. G., Semmler-Behnke, M., and Möller, W. (2006). Ultrafine particle-lung interactions: Does size matter? Journal of Aerosol Medicine, 19(1):74-83.

[40] Kronenburg, A., Bilger, R. W., and Kent, J. H. (2000). Modeling soot formation in turbulent methane-air jet diffusion flames. Combustion and Flame, 121(1-2):24-40.

[41] Lee, S. M., Yoon, S. S., and Chung, S. H. (2004). Synergistic effect on soot formation in counterflow diffusion flames of ethylene-propane mixtures with benzene addition. Combustion and Flame, 136(4):493-500.

[42] Leszczyszyn, O. (2012). https://www.materials-talks.com/blog/2012/11/15/size-matters-rh-versus-rg/. 
[43] Lindstedt, R. P. and Louloudi, S. A. (2005). Joint-scalar transported PDF modeling of soot formation and oxidation. Proceedings of the Combustion Institute, 30(1):775-783.

[44] Link, O., Snelling, D. R., Thomson, K. A., and Smallwood, G. J. (2011). Development of absolute intensity multi-angle light scattering for the determination of polydisperse soot aggregate properties. Proceedings of the Combustion Institute, 33(1):847-854.

[45] Liu, A. and Rigopoulos, S. (2019). A conservative method for numerical solution of the population balance equation, and application to soot formation. Combustion and Flame, 205:506-521.

[46] Liu, F., Guo, H., Smallwood, G., and Gülder, Ö. (2003). Numerical modelling of soot formation and oxidation in laminar coflow non-smoking and smoking ethylene diffusion flames. Combustion Theory and Modelling, 7(2):301-315.

[47] Neuber, G., Kronenburg, A., Stein, O. T., Garcia, C. E., Williams, B. A., Beyrau, F., and Cleary, M. J. (2020). Sparse-Lagrangian PDF modelling of silica synthesis from silane jets in vitiated co-flows with varying inflow conditions. Flow, Turbulence and Combustion.

[48] Patankar, S. V. (1980). Numerical Heat Transfer and Fluid Flow. Hemisphere Publishing Corporation, New York.

[49] Pope, S. B. (1985). PDF methods for turbulent reactive flows. Progress in Energy and Combustion Science, 11(2):119-192.

[50] Pratsinis, S. E. (1988). Simultaneous nucleation, condensation, and coagulation in aerosol reactors. Journal of Colloid and Interface Science, 124(2):416-427.

[51] Qamar, S., Elsner, M. P., Angelov, I. A., Warnecke, G., and Seidel-Morgenstern, A. (2006). A comparative study of high resolution schemes for solving population balances in crystallization. Computers \& Chemical Engineering, 30(6-7):1119-1131.

[52] Raj, A., Sander, M., Janardhanan, V., and Kraft, M. (2010). A study on the coagulation of polycyclic aromatic hydrocarbon clusters to determine their collision efficiency. Combustion and Flame, 157(3):523-534.

[53] Raman, V. and Fox, R. O. (2016). Modeling of fine-particle formation in turbulent flames. Annual Review of Fluid Mechanics, 48:159-190.

[54] Reimann, J., Kuhlmann, S.-A., and Will, S. (2009). 2D aggregate sizing by combining laser-induced incandescence (LII) and elastic light scattering (ELS). Applied Physics B, 96(4):583-592.

[55] Rigopoulos, S. (2010). Population balance modelling of polydispersed particles in reactive flows. Progress in Energy and Combustion Science, 36(4):412-443.

[56] Rigopoulos, S. (2019). Modelling of soot aerosol dynamics in turbulent flow. Flow, Turbulence and Combustion, 103(3):565-604.

[57] Sandkühler, P., Sefcik, J., and Morbidelli, M. (2004). Kinetics of aggregation and gel formation in concentrated polystyrene colloids. Journal of Physical Chemistry B, 108(52):20105-20121.

[58] Santoro, R. J., Semerjian, H. G., and Dobbins, R. A. (1983). Soot particle measurements in diffusion flames. Combustion and Flame, 51:203-218.

[59] Santoro, R. J., Yeh, T. T., Horvath, J. J., and Semerjian, H. G. (1987). The transport and growth of soot particles in laminar diffusion flames. Combustion Science and Technology, 53(2-3):89-115.

[60] Seitzman, J. M. and Hanson, R. K. (1993). Comparison of excitation techniques for quantitative fluorescence imaging of reacting flows. AIAA Journal, 31(3):513-519.

[61] Sorensen, C. M. (2013). Q-space analysis of scattering by particles: A review. Journal of Quantitative Spectroscopy and Radiative Transfer, 131:3-12. 
[62] Suzuki, S., Kuwana, K., and Dobashi, R. (2009). Effect of particle morphology on thermophoretic velocity of aggregated soot particles. International Journal of Heat and Mass Transfer, 52(21):4695-4700.

[63] Tamura, M., Berg, P. A., Harrington, J. E., Luque, J., Jeffries, J. B., Smith, G. P., and Crosley, D. R. (1998). Collisional quenching of $\mathrm{CH}(\mathrm{A}), \mathrm{OH}(\mathrm{A})$, and $\mathrm{NO}(\mathrm{A})$ in low pressure hydrocarbon flames. Combustion and Flame, 114(3):502-514.

[64] Tian, B., Gao, Y., Balusamy, S., and Hochgreb, S. (2015). High spatial resolution laser cavity extinction and laser-induced incandescence in low-soot-producing flames. Applied Physics B, 120(3):469-487.

[65] Tian, K., Thomson, K. A., Liu, F., Snelling, D. R., Smallwood, G. J., and Wang, D. (2006). Determination of the morphology of soot aggregates using the relative optical density method for the analysis of TEM images. Combustion and Flame, 144(4):782-791.

[66] Toda, A., Ohi, Y., Dobashi, R., Hirano, T., and Sakuraya, T. (1996). Accurate measurement of thermophoretic effect in microgravity. Journal of Chemical Physics, 105(16):7083-7087.

[67] Vemury, S. and Pratsinis, S. E. (1995). Self-preserving size distributions of agglomerates. Journal of Aerosol Science, 26(2):175-185.

[68] Veshkini, A., Dworkin, S. B., and Thomson, M. J. (2014). A soot particle surface reactivity model applied to a wide range of laminar ethylene/air flames. Combustion and Flame, 161(12):3191-3200.

[69] Waldmann, L. (1961). On the motion of spherical particles in nonhomogeneous gases. In Talbot, L., editor, Rarefied gas dynamics. Proceedings of the Second International Symposium on Rarefied Gas Dynamics, Held at the University of California, Berkeley, California, in 1960, pages 323-344. Academic Press, New York, London.

[70] Wang, H. (2011). Formation of nascent soot and other condensed-phase materials in flames. Proceedings of the Combustion Institute, 33(1):41-67.

[71] Wang, H. and Frenklach, M. (1997). A detailed kinetic modeling study of aromatics formation in laminar premixed acetylene and ethylene flames. Combustion and Flame, 110(1):173-221.

[72] Wang, Y., Raj, A., and Chung, S. H. (2013). A PAH growth mechanism and synergistic effect on PAH formation in counterflow diffusion flames. Combustion and Flame, 160(9):1667-1676.

[73] Wang, Y., Raj, A., and Chung, S. H. (2015). Soot modeling of counterflow diffusion flames of ethylene-based binary mixture fuels. Combustion and Flame, 162(3):586-596.

[74] Woods, I. T. and Haynes, B. S. (1991). Soot surface growth at active sites. Combustion and Flame, 85(3):523525 .

[75] Yapp, E. K. Y., Wells, C. G., Akroyd, J., Mosbach, S., Xu, R., and Kraft, M. (2017). Modelling PAH curvature in laminar premixed flames using a detailed population balance model. Combustion and Flame, 176:172-180.

[76] Yon, J., Lemaire, R., Therssen, E., Desgroux, P., Coppalle, A., and Ren, K. F. (2011). Examination of wavelength dependent soot optical properties of diesel and diesel/rapeseed methyl ester mixture by extinction spectra analysis and LII measurements. Applied Physics B, 104(2):253-271.

[77] Yunardi, Y., Darmadi, D., Hisbullah, H., and Fairweather, M. (2011). Investigation of detailed kinetic scheme performance on modelling of turbulent non-premixed sooting flames. Journal of Thermal Science, 20(6):548555. 


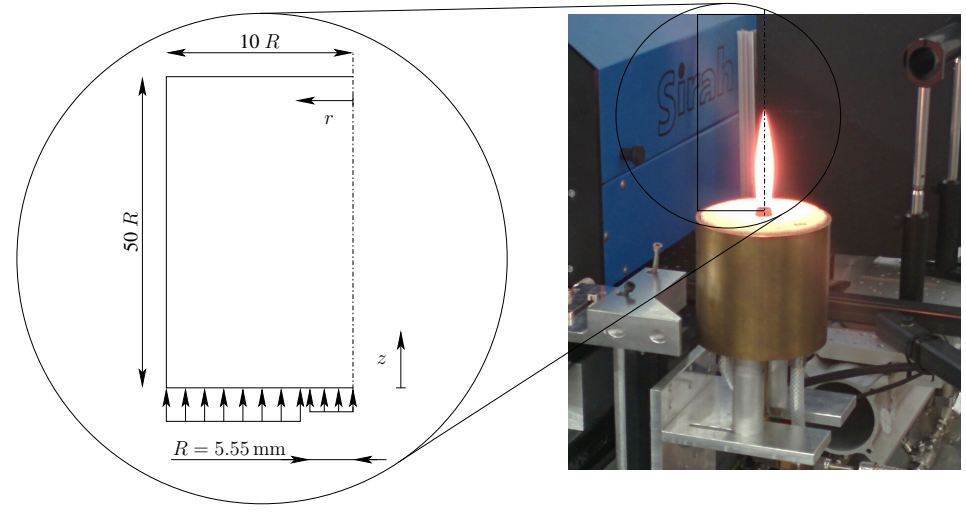

Figure 1: Schematic illustration of the computational domain (left) and photograph of the non-smoking flame on Santoro's co-flow burner without the shrouding chimney (right).

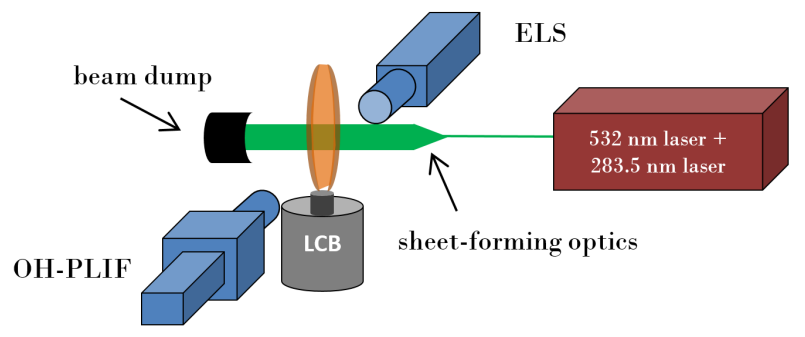

Figure 2: Schematic of the laser diagnostic setup. The $532 \mathrm{~nm}$ and $283.5 \mathrm{~nm}$ beams are coincident above the laminar co-flow burner (LCB). 

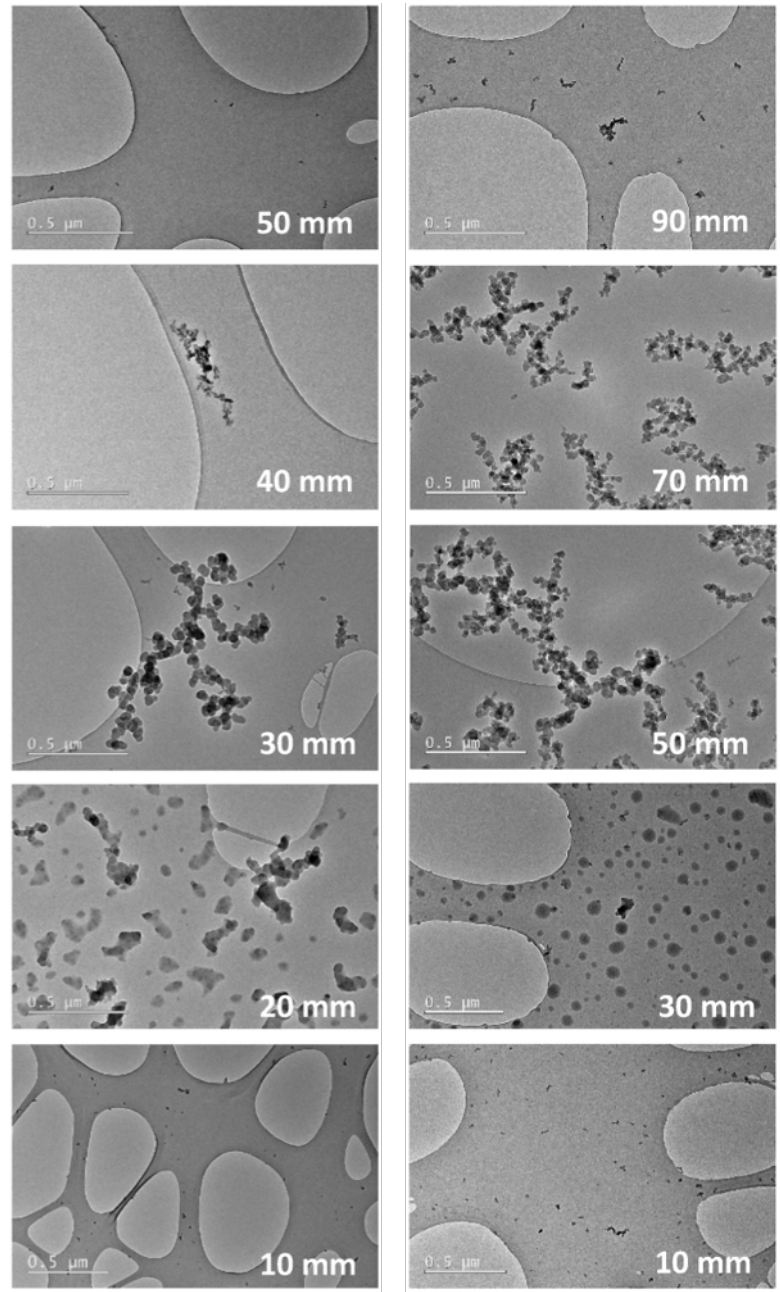

Non-smoking flame

Incipient-smoking flame

Figure 3: TEM micrographs of thermophoretically sampled soot particles on the centreline in the nonsmoking and incipient-smoking flames. Each image covers approximately $1.5 \times 2 \mu \mathrm{m}$ and soot particles are supported on a holey carbon film. 


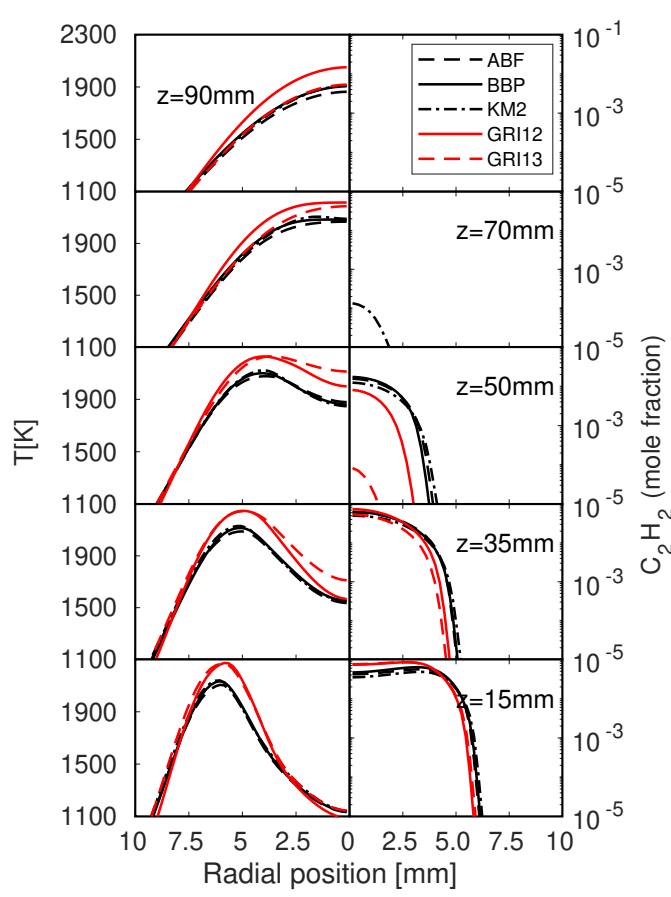

(a) Temperature and $\mathrm{C}_{2} \mathrm{H}_{2}$ mole fraction

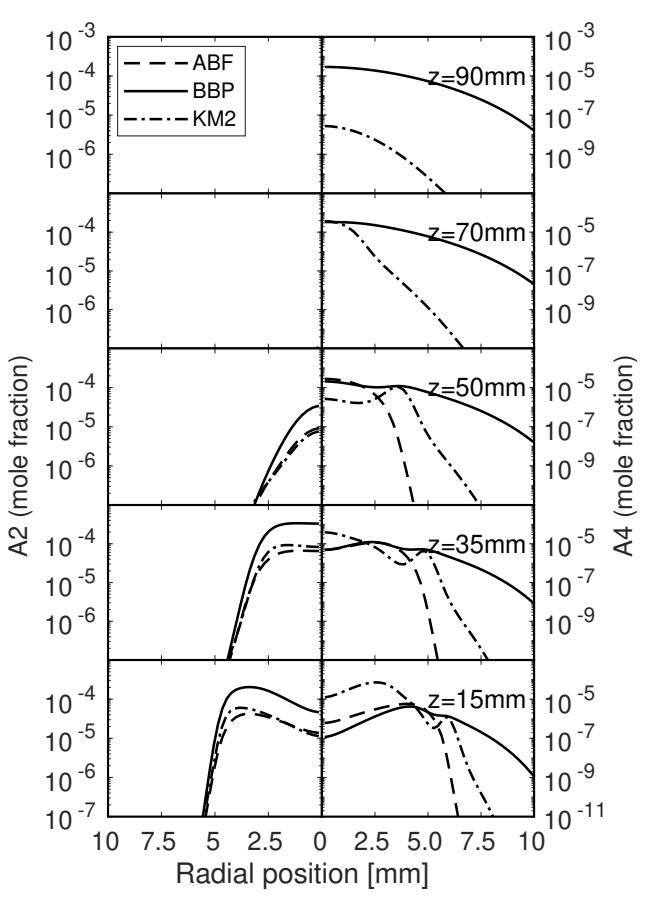

(b) $\mathrm{A}_{2}$ and $\mathrm{A}_{4}$ mole fractions

Figure 4: Comparison of the temperature and the mole fractions of $\mathrm{C}_{2} \mathrm{H}_{2}$ and the PAHs $\mathrm{A} 2$ and $\mathrm{A} 4$ for the three PAH-based reaction mechanisms ABF, BBP and KM2 and the non-PAH-baseline mechanisms GRI 1.2 and GRI 3.0 in the hot flow of the incipient-smoking flame when soot formation is deactivated.

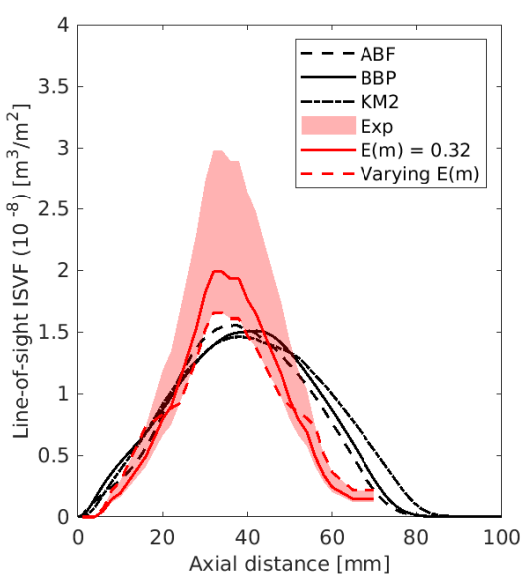

(a) Non-smoking flame

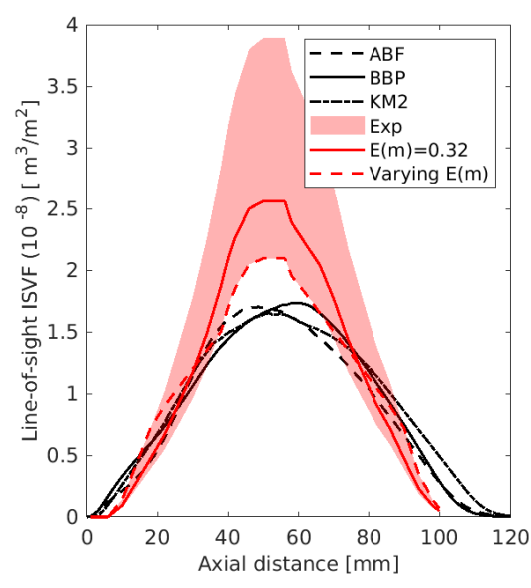

(b) Incipient-smoking flame

Figure 5: Comparison between the predicted line-of-sight ISVF along the flame centreline and the ISVF obtained from discrete LOSA measurements. 


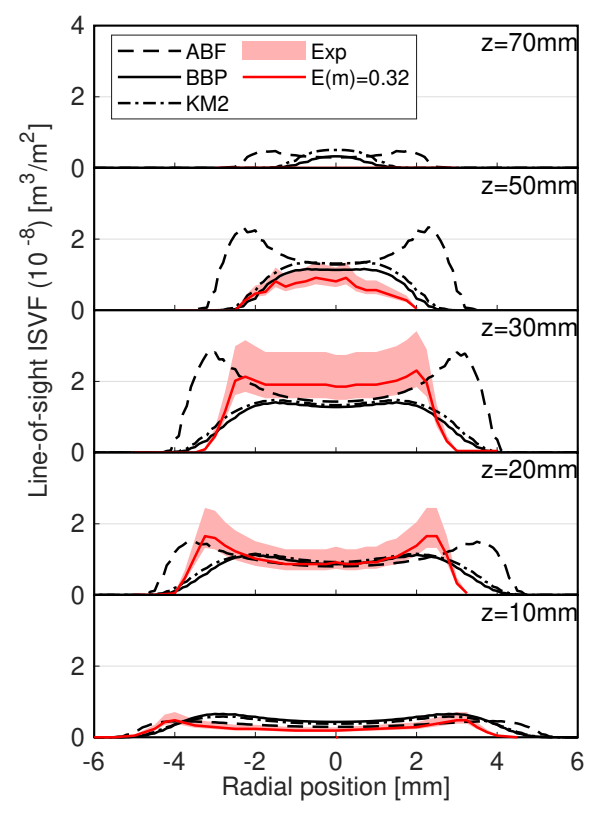

(a) Non-smoking flame

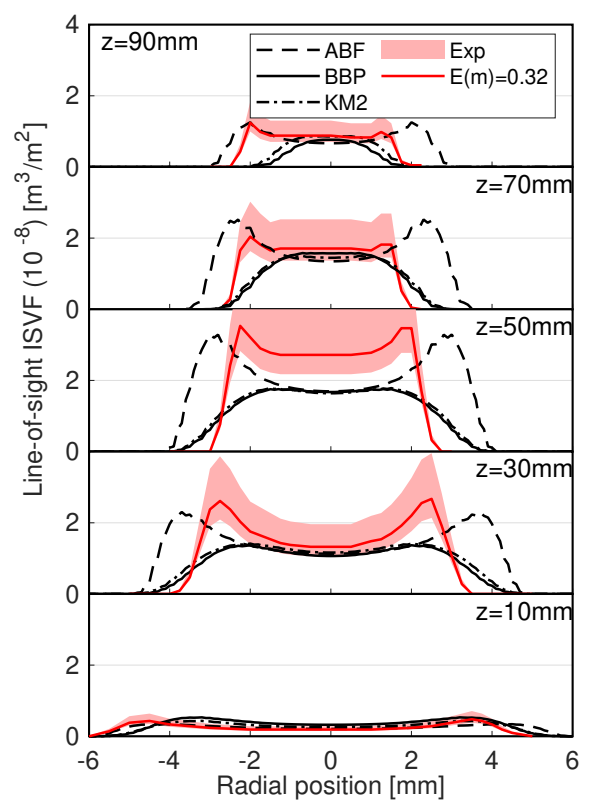

(b) Incipient-smoking flame

Figure 6: Comparison of predicted and measured radial profiles of the line-of-sight ISVF.

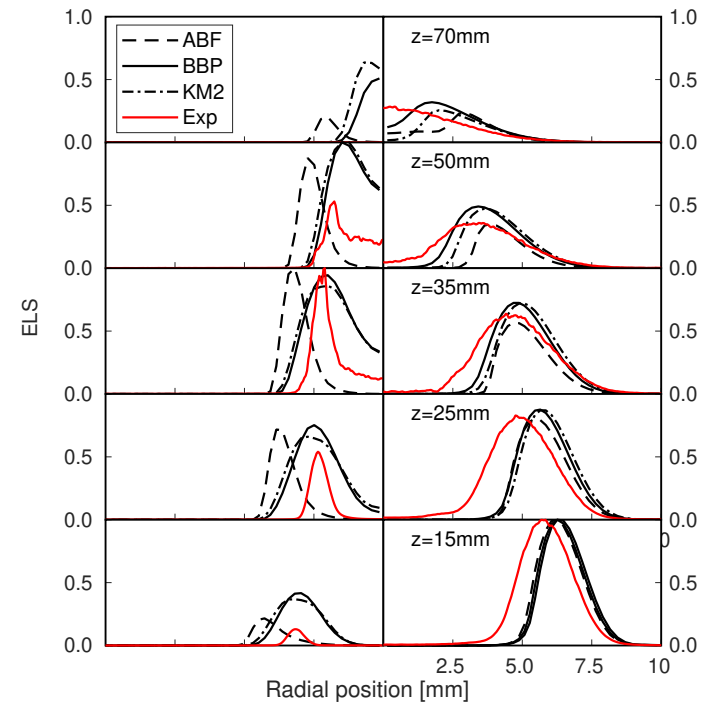

(a) Non-smoking flame

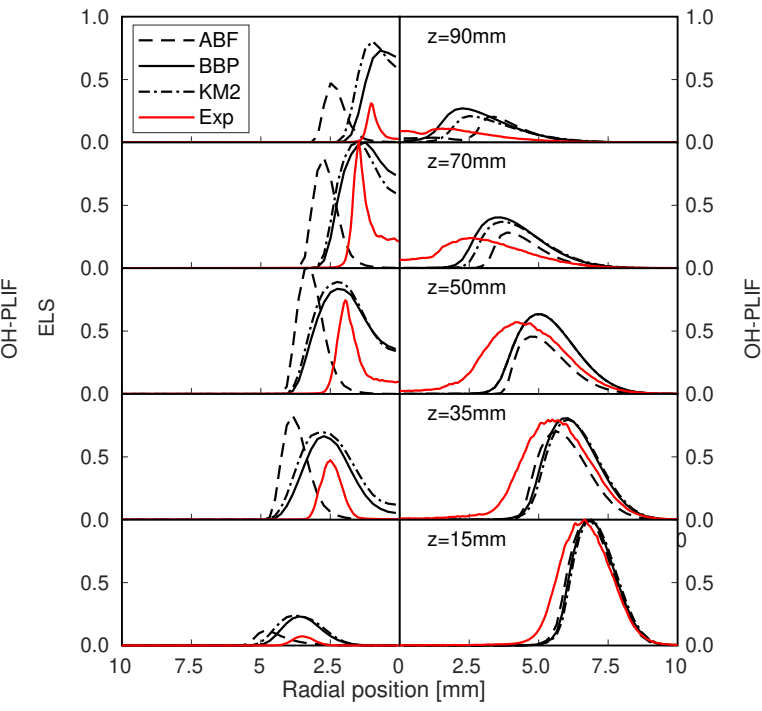

(b) Incipient-smoking flame

Figure 7: Radial profiles of the ELS and OH-PLIF signals at different axial locations for the non-smoking and incipient-smoking flames. 


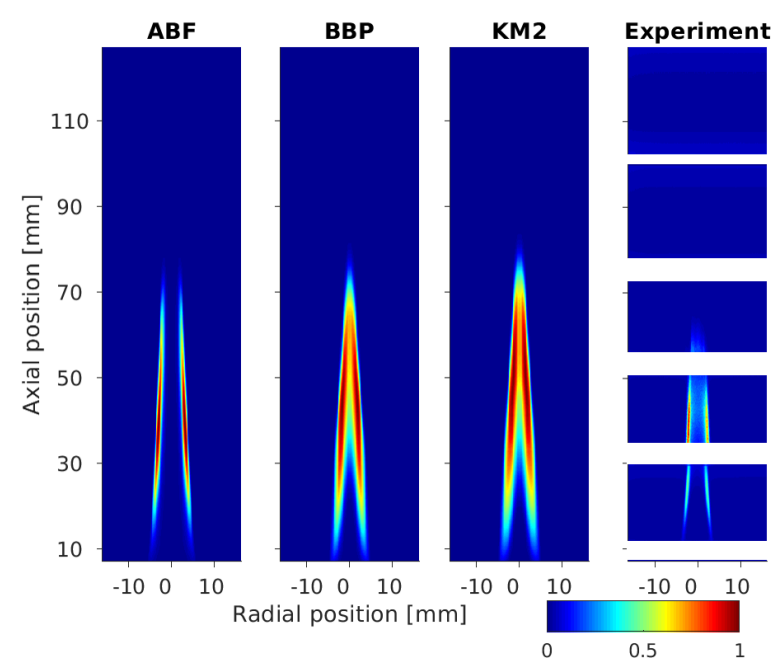

(a) ELS signals

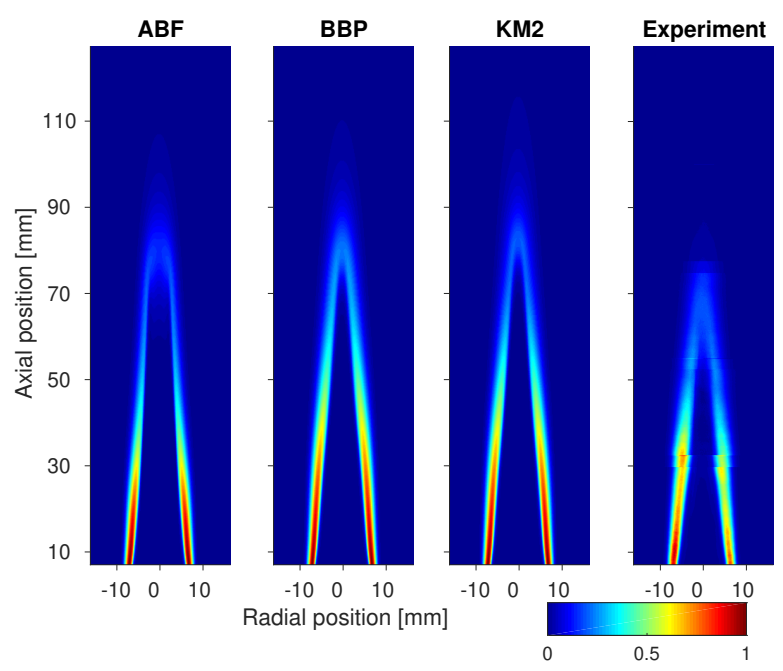

(b) OH-PLIF signals

Figure 8: Contour plots of the ELS and OH-PLIF signals at different downstream locations in the nonsmoking flame. The white stripes in the ELS experimental data correspond to regions where data is not available.

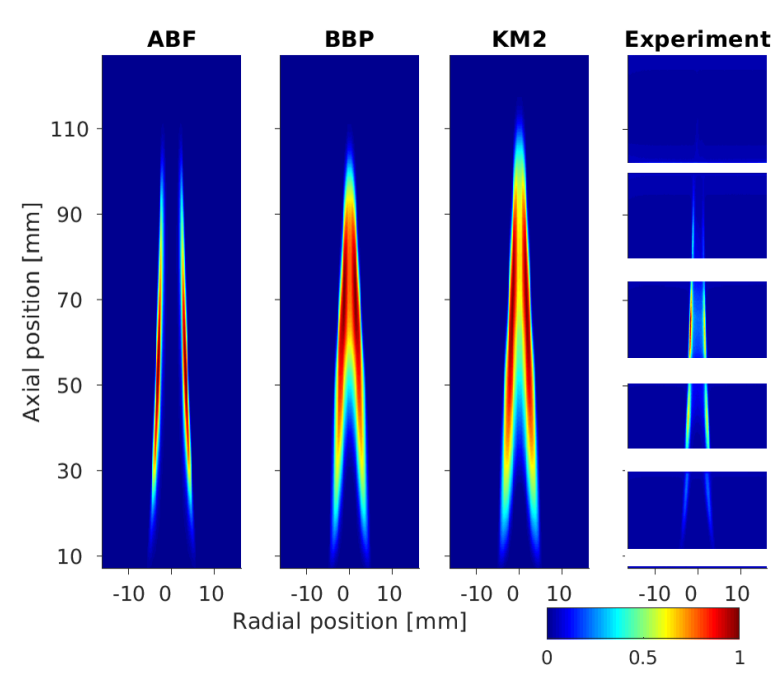

(a) ELS signals

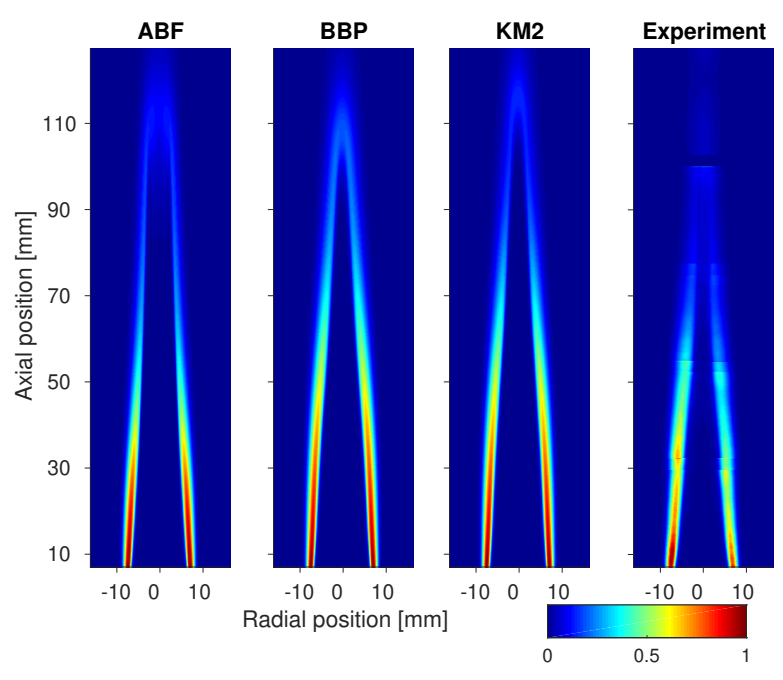

(b) OH-PLIF signals

Figure 9: Contour plots of the ELS and OH-PLIF signals at different downstream locations in the incipientsmoking flame. The white stripes in the ELS experimental data correspond to regions where data is not available. 


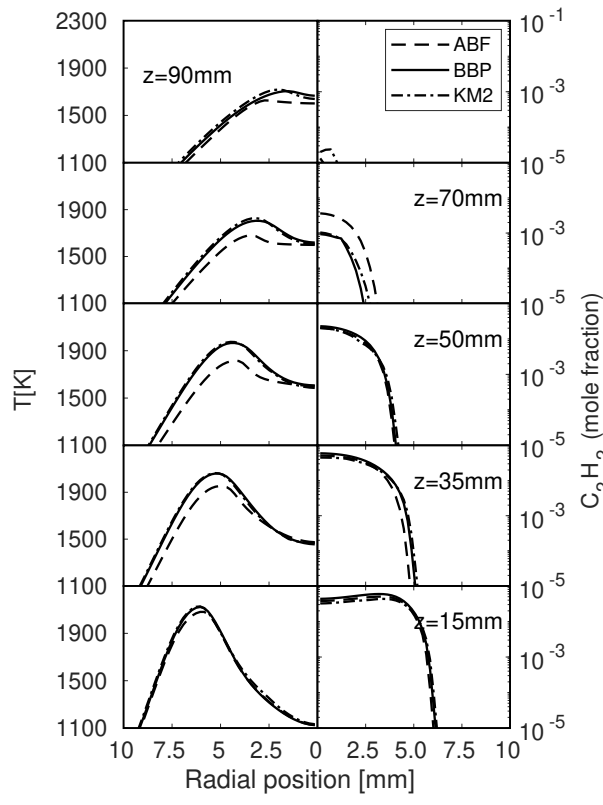

(a) Temperature and $\mathrm{C}_{2} \mathrm{H}_{2}$ mole fraction

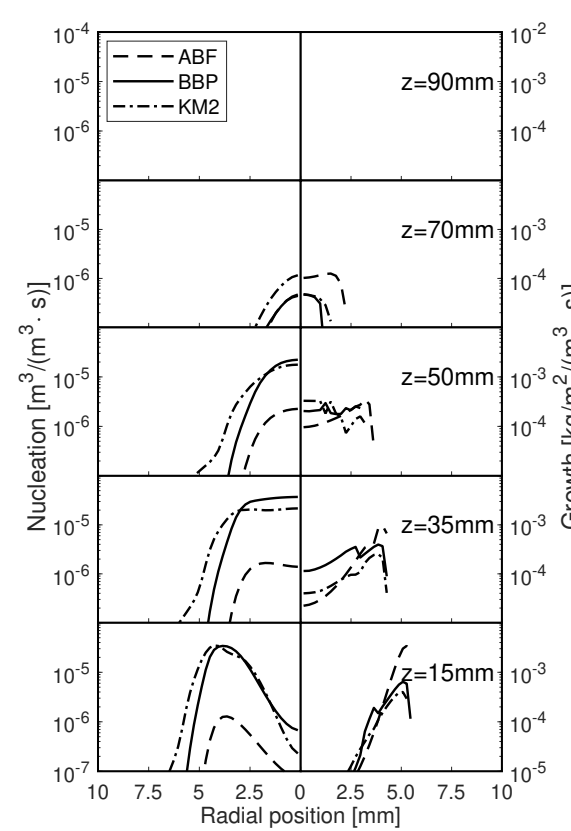

(c) Nucleation rate and HACA surface growth (d) Oxidation rate and PAH condensation rate

rate

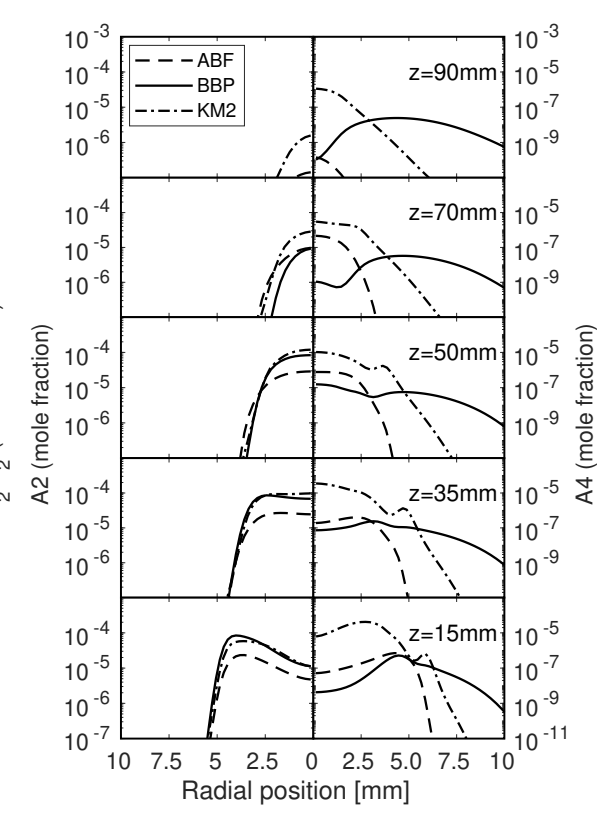

(b) $\mathrm{A}_{2}$ mole fraction and $\mathrm{A}_{4}$ mole fraction

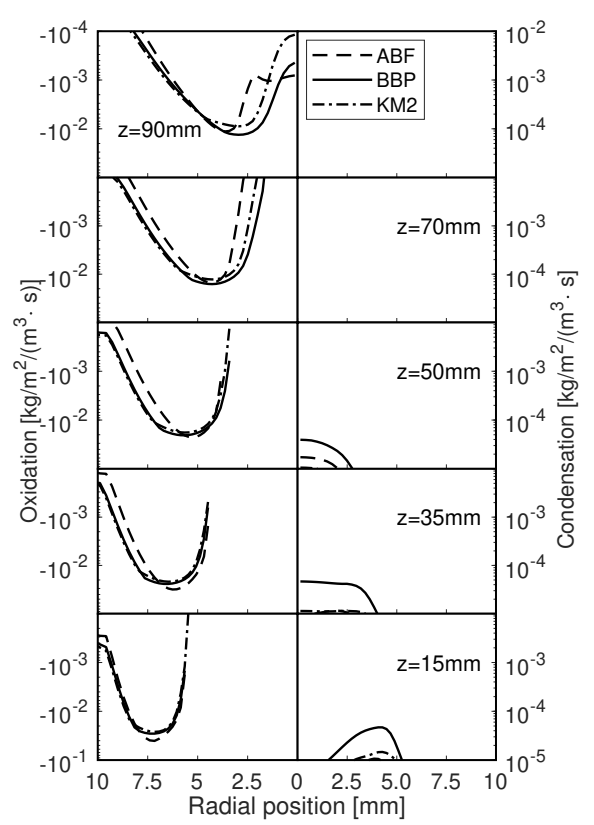

Figure 10: Radial profiles of key variables controlling soot formation at different downstream locations in the incipient-smoking flame. 


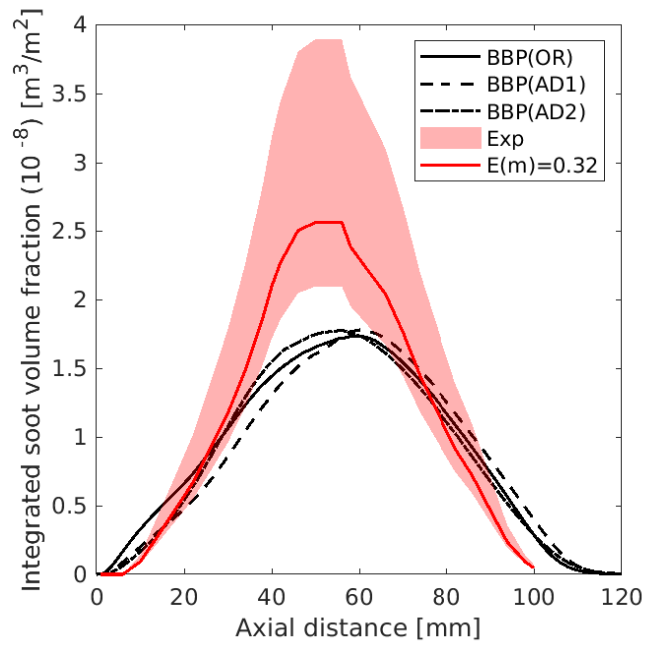

Figure 11: Comparison between experimental measurements and line-of-sight ISVFs predicted by the three parameter sets listed in Table 4.

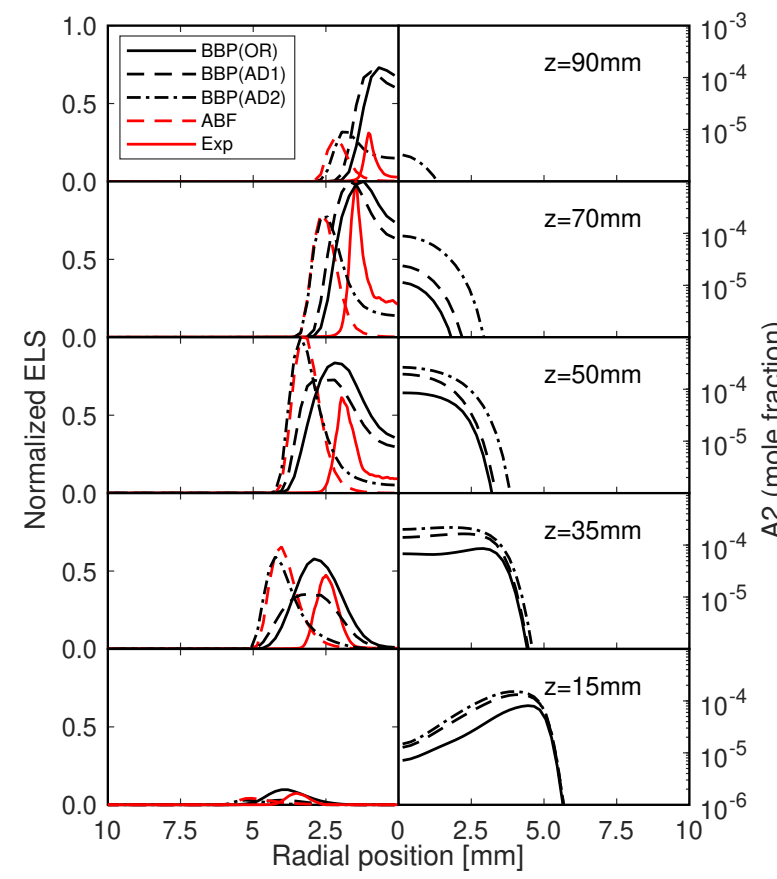

(a) ELS signal (left) and $\mathrm{A}_{2}$ mole fraction (right)

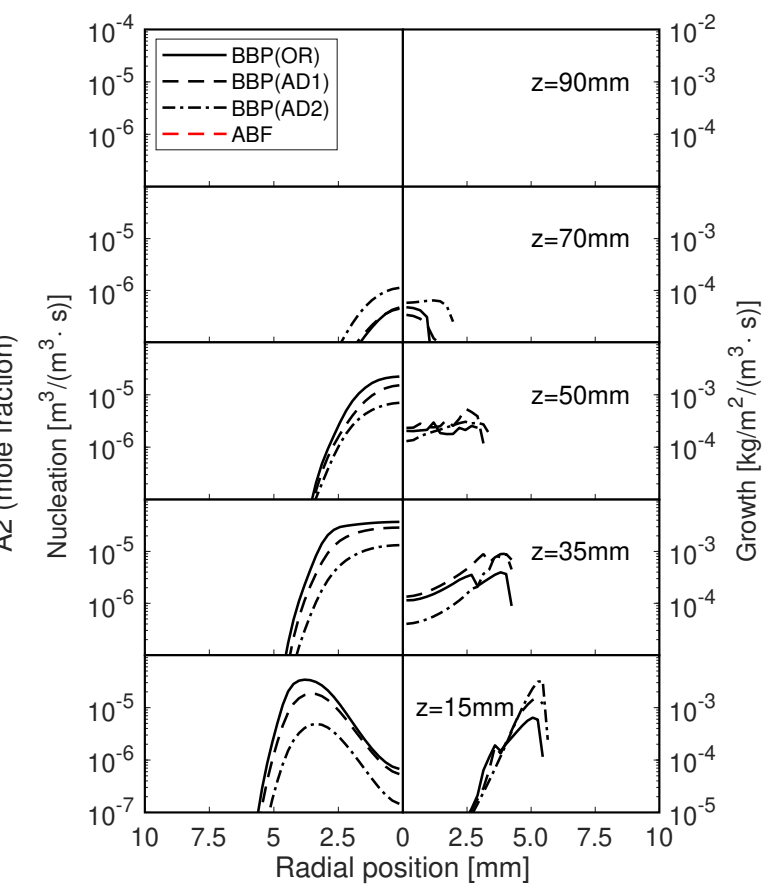

(b) Nucleation rate (left) and HACA surface growth rate (right)

Figure 12: Radial profiles of key variables for the sensitivity analysis in terms of nucleation/condensation and growth rates based on the parameter sets in Table 4 